\title{
Incipient thermal choking and stable shock-train formation in the heat-release region of a scramjet combustor. Part II: Large eddy simulations
}

\author{
Johan Larsson $^{\mathrm{a}, *}$, Stuart Laurence ${ }^{\mathrm{b}}$, Ivan Bermejo-Moreno ${ }^{\mathrm{a}}$, Julien Bodart ${ }^{\mathrm{a}, 1}$, Sebastian Karl ${ }^{\mathrm{c}}$, Ronan \\ Vicquelin ${ }^{\mathrm{d}}$ \\ ${ }^{a}$ Center for Turbulence Research, Stanford University \\ ${ }^{b}$ Dept. of Aerospace Engineering, University of Maryland \\ ${ }^{c}$ Institute of Aerodynamics and Flow Technology, Spacecraft Dept., German Aerospace Center (DLR) \\ ${ }^{d}$ Ecole Centrale Paris
}

\begin{abstract}
The flow in the HyShot II scramjet combustor is studied using large eddy simulations (LES). The computations are made feasible by two important modeling ingredients: an equilibrium wall-model and a flamelet-based combustion model. The first objective of the study is to assess the accuracy of this modeling approach through a validation study. Comparisons are made between simulation results and those from shock-tunnel experiments at nominal flow conditions, with favorable agreement. The second objective is to study the flow for increased fuel/air equivalence ratios (ERs). A qualitative change in the flow occurs for ER $\gtrsim 0.39$, with the appearance of a seemingly stable combustor shock-train, similar to standard isolator shock-trains, but occurring spatially co-located with the combustion and heat release. This behavior accurately reproduces that seen in an accompanying experimental study. A detailed flow analysis identifies the factors contributing to the stabilization of the shock-train, and estimates are made of its effect on the overall combustor performance.
\end{abstract}

Keywords:

\section{Introduction}

The supersonic combustion ramjet (scramjet) is a theoretically efficient means of propulsion for atmospheric flight at Mach numbers above about 5. Despite the simplicity of the scramjet concept, however, there are major technical challenges associated with developing a working scramjet-powered vehicle. As the core airflow remains supersonic throughout the engine flowpath, injection, mixing, and combustion of the fuel must take place on very short time-scales. Moreover, since a high-speed air-breathing vehicle

\footnotetext{
${ }^{*}$ Present address: Dept. of Mechanical Engineering, University of Maryland

Email address: jola@umd.edu (Johan Larsson)

${ }^{1}$ Present address: Université Toulouse, ISAE
} 
must operate over a wide range of Mach numbers, to reduce system complexity it is highly desirable that the scramjet-based engine component exhibits good performance throughout this spectrum of conditions, not just at the high Mach numbers to which a pure scramjet is best suited [1].

This latter requirement has led to the development of the dual-mode concept [2], in which an isolator (typically a constant-area diffusor) is introduced into the scramjet flowpath between the intake and the fuel-injection location. This isolator serves to house a precombustion shock structure when the engine is operating away from nominal scramjet (i.e., purely supersonic) conditions. Such a shock structure may be brought about in one of two ways $[3,1]$. First, at lower flight Mach numbers, the combustor heat release is tailored so that the flow becomes choked (i.e., the Mach number is reduced to unity), causing a normal shock-train with a subsonic core to form in the isolator (see Matsuo et al. [4] for a review of shock-train phenomena). In this way, the engine operates as a traditional ramjet, with the area change downstream tailored to re-accelerate the flow to supersonic conditions. As the flight Mach number increases, however, the stagnation pressure loss associated with a normal shock-train would reduce the cycle efficiency substantially; furthermore, the pressures and temperatures (the latter resulting in dissociation and a loss of available chemical energy) produced by decelerating the flow to subsonic conditions become increasingly undesirable $[3,5]$. Thus, in this regime the dual-mode engine operates in scramjet mode, with the core flow supersonic throughout. Nevertheless, it may still arise that the adverse pressure gradient inside the combustion chamber causes the wall boundary layers to separate, forming an oblique shock-train (with a supersonic core) that subsequently propagates upstream. In this (second) case, the isolator serves to confine this oblique shock-train, preventing it from propagating further and affecting the intake flow.

If the combustion-induced pressure rise is too large for the shock structure in the isolator to adapt to, or if no isolator is present, the shock-train (normal or oblique) will propagate further upstream, leading to inlet unstart [1]. Unstart, defined as the upstream displacement or "disgorging" of the original inlet shock system, is highly undesirable. The resulting flow spillage reduces the engine performance; moreover, the detached shock that forms can be highly unsteady, generating violent loads on the vehicle [3]. Therefore, an understanding of the fluid-combustion phenomena responsible for the formation of the shock structures that can lead to unstart is crucial for the reliable operation and robust design of scramjet-powered vehicles.

\subsection{The role of LES for scramjets}

Predictive simulations have a large role to play in the development of scramjet technology. At flight conditions, the effective free-stream stagnation pressure can be of the order of tens of MPa and the stagnation temperature well in excess of $1000 \mathrm{~K}$. Realistic ground-testing at such conditions is extremely challenging, and all approaches lead to some form of limitation. Pre-heating the incoming flow using vitiation- or arc-heating provides long test times, but also introduces unwanted constituents in the oxidizer flow (see Ref. [3], p. 535). In general, this modified chemical composition will aid the ability of the fuel to ignite and burn, thus not representing the flight conditions exactly. Shock tunnels and expansion tunnels provide clean incoming air, but typically have test times limited to a few milliseconds.

Although steady-state simulation methods (most obviously, RANS) may often yield sufficiently accurate predictions for design and assessment at steady operating conditions, it is well known that these 
methods are less trustworthy in the presence of large-scale unsteady and/or separated flow. The large eddy simulation (LES) technique is generally more accurate for such flows, and also typically provides more accurate predictions of the turbulent mixing process. LES can therefore be expected to yield more accurate and trustworthy predictions than RANS (cf. Fulton et al. [6] for a direct comparison using a relevant scramjet flow). The major problem is that standard LES would require a completely infeasible computational cost if applied to a scramjet engine. The only feasible approach is to use LES with a wallmodel, where the innermost $10-20 \%$ of the boundary layer is modeled; this reduces the computational cost by approximately three orders of magnitude for the combustor studied in the present work, for example.

A summary of the current state-of-the-art and different approaches in LES of scramjet flows is provided by Fureby [7].

\subsection{The HyShot II scramjet}

The HyShot II flight experiment was launched in 2002, successfully demonstrating supersonic combustion over a range of altitudes [8]. It was later the subject of multiple experimental investigations in the High Enthalphy shock tunnel Göttingen (HEG) of the German Aerospace Center (DLR) [9, 10, 11, $12,13,14,15]$. The flow in the HyShot II combustor has been studied computationally by at least three different groups. Karl et al. [16, 17, 18, 19, 20] performed a comprehensive RANS investigation of the full experimental set-up in the HEG shock tunnel, including the flow in the shock tunnel nozzle, the flow over the HyShot II forebody, and the reacting flow in the combustor. One outcome of this work was to show that the flow over the forebody can be accurately modeled as two-dimensional, a fact which is used in the present study. The chemically reacting flow was modeled Karl et al. by solving transport equations for the 9 species $\left(\mathrm{H}_{2}, \mathrm{O}_{2}, \mathrm{~N}_{2}, \mathrm{H}_{2} \mathrm{O}, \mathrm{H}, \mathrm{O}, \mathrm{OH}, \mathrm{HO}_{2}, \mathrm{H}_{2} \mathrm{O}_{2}\right)$ considered in the chemical mechanism. The main challenge in this approach is the closure of the chemical source terms; this was done by a presumed probability density function (PDF) approach, with both $\delta$ - and $\beta$-PDFs considered. While the latter was found to produce a pressure profile that agreed slightly better with the experimental one, the difference was found to be small.

Pecnik et al. [21] extended the classic flamelet-based modeling approach to the supersonic regime, and applied this model to the HyShot II combustor within a RANS framework. One important contribution of Pecnik et al. [21] was to assess the importance of the spanwise domain size on the RANS results. Specifically, they compared cases covering $1 / 2$ and $1 / 8$ of the combustor width using the appropriate symmetry boundary conditions, and showed that the differences in the results were small. This finding will be used in the present study to model only a single injector in the LES, i.e., 1/4 of the full width.

Fureby et al. [22] and later Chapuis et al. [23] simulated the HyShot II combustor flow using LES. They solved transport equations for 7 species with a partially stirred reactor model to account for the unresolved chemical reaction fronts. In the earlier work [22] only half of the combustor width was modelled, with a symmetry boundary condition along the centerline. In the follow-on work [23], the full width of the combustor was included in the computation, using a grid of $51 \mathrm{M}$ cells.

We note that the reactive flow in the HyShot II combustor is primarily mixing-controlled under the conditions considered here, with the possible exception of the flame-anchoring near the fuel injector. Berglund and Fureby [24] estimated a Damköhler number of $\sim 40$ in a (different) scramjet combustor with 
cold $(340 \mathrm{~K})$ incoming air. The incoming air in the present case is at $1300 \mathrm{~K}$ which leads to correspondingly faster chemistry. When using the present LES results to estimate a turbulent time scale representative of the combustor, we arrive at a Damköhler number of order $\mathcal{O}(100)$. Therefore, a flamelet-based combustion model seems justified. More importantly, in a mixing-controlled flow, details of the combustion model have less influence on the solution. The relative agreement between the computed pressure profiles by Karl et al. [19] and Pecnik et al. [21] (two studies which used very different turbulent combustion models) is consistent with this.

\subsection{Objectives}

The aim of the present study is twofold. First, we assess the ability of a wall-modeled LES method with a flamelet-based combustion model to accurately predict the flow in the HyShot II scramjet combustor at a manageable computational cost. Simulations are compared to earlier experimental data from HEG. Second, we apply this LES methodology to cases at higher equivalence ratios, both to predict the critical equivalence ratio at which unstart-like phenomena are first observed in the HyShot II combustor and to study and characterize the flow at these conditions. The predictions are compared with experiments carried out for the same purpose in HEG, described in the companion paper [15].

\section{Methodology}

\subsection{LES methodology}

The filtered compressible Navier-Stokes equations are solved for the conserved variables. The total energy $E$ is defined as the sum of sensible, kinetic and chemical energies. The residual subgrid stress is modeled using an eddy-viscosity hypothesis together with the model by Vreman [25]. The subgrid heat flux and species transport are modeled using gradient hypotheses with fixed turbulent Prandtl and Schmidt numbers, both taken as 0.5. The equations are implemented in the unstructured code CharlesX, which uses a solution-adaptive approach mathematically analyzed in a set of papers [26, 27] in which a background scheme with low aliasing and dissipation errors is used away from shock waves while an unstructured essentially non-oscillatory (ENO) second-order accurate shock-capturing scheme with an HLLC approximate Riemann solver [28] is applied near discontinuities. The background scheme is only non-dissipative for perfectly regular grids, but adds a small amount of numerical dissipation on irregular grids to aid the robustness. Further description of the code can be found in Khalighi et al. [29].

At each time step, the shock-capturing scheme is applied if the negative rate of dilatation $-\partial_{j} u_{j}>$ $\max \left(\sqrt{\omega_{j} \omega_{j}}, 0.1 c / h\right)$, where $\omega_{j}$ is the vorticity and $c / h$ is the speed-of-sound divided by the cell size. The shock-capturing scheme is additionally applied if two adjacent cells differ by more than $500 \mathrm{~K}$ in temperature, by more than 0.4 in the mixture fraction, or by more than 0.2 in the mass fraction of $\mathrm{H}_{2} \mathrm{O}$. Given the low numerical dissipation of the background numerics, the exact form of the sensor is rather important in order to ensure robust results. The inclusion of the speed-of-sound in the threshold for the dilatation is necessary to avoid activating the sensor in a bulk flow, or in a free stream. This could have detrimental effects on the accuracy of capturing acoustic waves, especially in an unstructured code where 


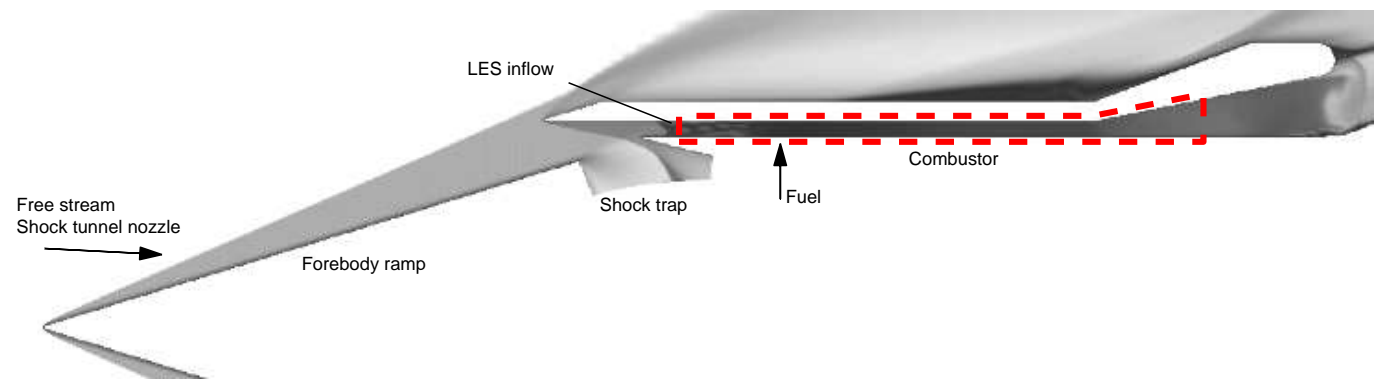

Figure 1: A slice through the HyShot II vehicle. The free stream (or flow from the shock tunnel nozzle) approaches at a slight angle-of-attack of $3.6^{\circ}$. The flow passes through the strong bow shock and the shock from the upper combustor wall; this shock is then swallowed by a shock-trap, leading to a relatively weak shock system in the isolator. The LES is performed in a domain covering the combustor and parts of the isolator and nozzle, respectively (shown with the dashed line). The inflow conditions to the LES domain are computed through an auxiliary 2D RANS computation, covering the full domain shown in the figure.

the shock-capturing numerics are generally of lower order in accuracy. On the medium grid used in this study, about $3-4 \%$ of all faces are treated using the shock-capturing ENO/HLLC scheme.

The simulations are run for up to $10 \mathrm{~ms}$, which is longer than the actual test time in the shock tunnel. About $200 \cdot 10^{3}, 80 \cdot 10^{3}$, and $16 \cdot 10^{3}$ core-hours are needed to advance the solution by $1 \mathrm{~ms}$ on the three grids (to be discussed later), respectively, on a cluster with Intel Westmere processors. The most expensive calculation, on the medium grid at equivalence ratio $\mathrm{ER}=0.414$, thus required almost one million core-hours.

\subsection{Domains and inflow conditions}

The geometry of the HyShot II vehicle can be seen in Figs. 1 and 2. The isolator/combustor section is $75 \mathrm{~mm}$ wide and $9.8 \mathrm{~mm}$ high. Pure hydrogen fuel is injected through four equi-distantly spaced injectors located $57.5 \mathrm{~mm}$ downstream of the lip of the lower wall. The end of the combustor and the beginning of the nozzle is located $242.5 \mathrm{~mm}$ downstream of the fuel injectors. The forebody ramp (between the vehicle nose and the entrance to the isolator) is wider than the isolator/combustor to make the flow effectively two-dimensional there. The forebody boundary layer and the shock from the upper wall lip are bled in a shock-trap, thus causing a flow with only a weak shock system to enter the isolator.

An instantaneous snapshot from an LES at nominal conditions is shown in Fig. 2. The multiple incoming oblique shock waves are visible in the Schlieren image, as is the bow shock around the fuel jet. The fuel jet is initially relatively unperturbed, but breaks down into full turbulence farther downstream. The velocity contours show the thin incoming boundary layers and how these grow farther downstream. The temperature contour shows how the heat release leads to increased temperatures downstream, up to about $2500 \mathrm{~K}$ before the nozzle. Finally, note that the momentum flux ratio of the fuel jets is low in HyShot II (between 0.3 and 0.5 depending on operating condition), and thus the fuel jets are rather weak.

The HyShot II geometry is such that the flow is essentially two-dimensional all the way from the free stream up to the fuel injectors. The flow in the isolator (i.e., before the fuel injectors) is fully supersonic, 


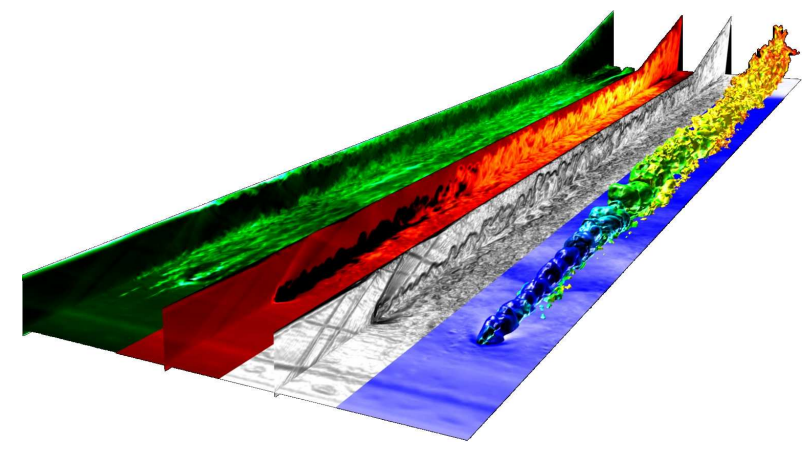

Figure 2: Instantaneous snapshot from LES of the HyShot II combustor. The computational domain (one injector) is replicated four times in the spanwise direction. From the left, the slices show contours of streamwise velocity $u_{1}$, temperature $T$, simulated Schlieren $|\nabla \rho|$, and pressure $p$. In addition, the right-most section shows an isosurface at the stoichiometric mixture fraction, colored by the $\mathrm{OH}$ concentration.

with the exception of only the very thin boundary layers. These two facts imply that a staggered solution procedure can be used, in which a 2D RANS is used to compute the flow up to the isolator, and where only the second part of the isolator and the combustor are modeled using 3D LES.

The auxiliary 2D RANS covers the full domain shown in Fig. 1. The RANS inflow conditions (corresponding to the conditions in the shock tunnel nozzle) are prescribed as follows. The total pressure and temperature $p_{0, \infty}$ and $T_{0, \infty}$ are measured for each run in the experiments. In the computations we use either values averaged over an experimental campaign or a run-specific value. From these conditions, the static values of $p_{\infty}$ and $T_{\infty}$ and the velocity $U_{\infty}$ are computed using relations for the shock tunnel nozzle computed using CFD at DLR $[19,11]$. The angle-of-attack is taken as $3.6^{\circ}$, the value used in the experiments. The turbulence intensity and length scale in the shock tunnel nozzle are estimated to be $1 \%$ (from unsteady pressure data) and $0.25 \mathrm{~m}$ (from the nozzle geometry), respectively. These values are very approximate, but have only a minor influence on the LES results since the forebody boundary layer is completely bled in the shock trap. The 2D RANS calculations were performed by Michael Emory as described in Emory et al. [30]. Note that the transition locations are specified from the experimental data; specifically, the boundary layer on the upper wall transitions well before the LES inlet, whereas the boundary layer on the lower wall remains laminar well into the isolator.

The computational domain for the LES is shown in Fig. 1. The LES domain starts $31 \mathrm{~mm}$ upstream of the center of the fuel injectors. This position was chosen since it is halfway between the first (off the upper wall) and second (off the lower wall) reflections of the shock from the lower combustor wall lip. Therefore, the main oblique shock entering the LES domain is halfway between the upper and lower walls at the chosen LES inlet location. The time-dependent turbulent inflow to the LES is specified from the mean RANS profiles together with the digital filtering technique of Klein et al. [31] to generate synthetic "turbulence". The walls are assumed isothermal at $300 \mathrm{~K}$ due to the short duration of the shock tunnel experiments.

To reduce the computational cost, only a single injector is included in the domain. Thus the com- 
putational domain is $1 / 4$ of the width of the full combustor, with periodic boundary conditions used in the spanwise direction. This approximation was investigated by Pecnik et al. [21] using RANS, and was found to be reasonable.

The fuel injector is modeled as a strong contraction, consistent with the true physical design. This results in a sonic, choked, fuel jet. The fuel injection rate, and thus the equivalence ratio (ER), is controlled by specifying the total fuel pressure $p_{0 \text {,fuel }}$. The total temperature of the fuel is always $300 \mathrm{~K}$.

The coordinate system used for the LES computations has its origin in the center of the fuel injector, level with the lower wall. Thus the streamwise $x$ coordinate is offset by $57.5 \mathrm{~mm}$ compared to the coordinates used in the experiments $[10,11,12,13,15]$ and RANS simulations $[16,17,18,19,20]$ performed at DLR.

\subsection{Combustion model}

A flamelet-based combustion model is used in this work since it leads to an affordable computational cost (no chemistry-induced stiffness) and since the estimated Damköhler number of order $\mathcal{O}(100)$ suggests that a flamelet approach is suitable. In addition, Saghafian [32] performed a DNS of a temporally evolving reacting supersonic mixing layer with the same chemical mechanism as in the present work, and found that the combustion was within the flamelet regime.

The steady flamelet-based model of Pecnik et al. [21], which is an extension of the steady flamelet/progressvariable model of Pierce and Moin [33] to supersonic flows, is used in this work. Laminar counterflow diffusion (non-premixed) flamelets are solved a priori at different strain-rates, with the results parametrized in terms of the mixture fraction $Z$ and the reaction progress-variable $C$, taken here as the mass fraction of $\mathrm{H}_{2} \mathrm{O}$. The effect of turbulence on the small-scale chemistry is modeled by a presumed $\beta$-PDF for the mixture fraction $Z$. For the reaction progress variable $C$, a $\delta$-PDF is assumed. Macro-scale (LES) transport equations are solved for the filtered mixture fraction $\widetilde{Z}$, the subfilter variance $\widetilde{Z^{\prime \prime} Z^{\prime \prime}}$ and the filtered progress variable $\widetilde{C}$. The results of the laminar flamelets are parametrized and tabulated in terms of these three scalars.

Flamelet-based models with a presumed PDF have been rather widely used in the area of subsonic combustion, but have been used much less for supersonic combustion. Sabelnikov et al. [34] studied the flamelet-concept for supersonic flows and how the large kinetic energies involved may change the flamelet solutions. Berglund and Fureby [24] used a flamelet/progress-variable approach in their LES of the flow around a wedge-shaped injector. Other studies have relied on other combustion modeling approaches. For example, the LES studies of the HyShot II scramjet by Fureby, Chapuis and co-workers [22, 23] solved transport equations for the species at the macro-level using a partially stirred reactor model to close the chemical source terms. Similarly, the LES of Edwards et al. [35] solved macro-level transport equations, but without any special closure for the source term. The chief difficulty in applying a flamelet model to supersonic combustion is the hydrodynamically induced variations in pressure and enthalpy (due to, e.g., shock waves, wall-cooling, etc). Since the flamelets are solved a priori with the results tabulated for later use in the LES, it is clear that there is a lack of feedback from the LES solver to the flamelet solver.

In the present work, an approach very similar to that proposed by Pecnik et al. [21] is followed. Flamelets are solved and tabulated at a single reference pressure of $p_{\text {tab }}=1.5$ bar, and at fixed tem- 

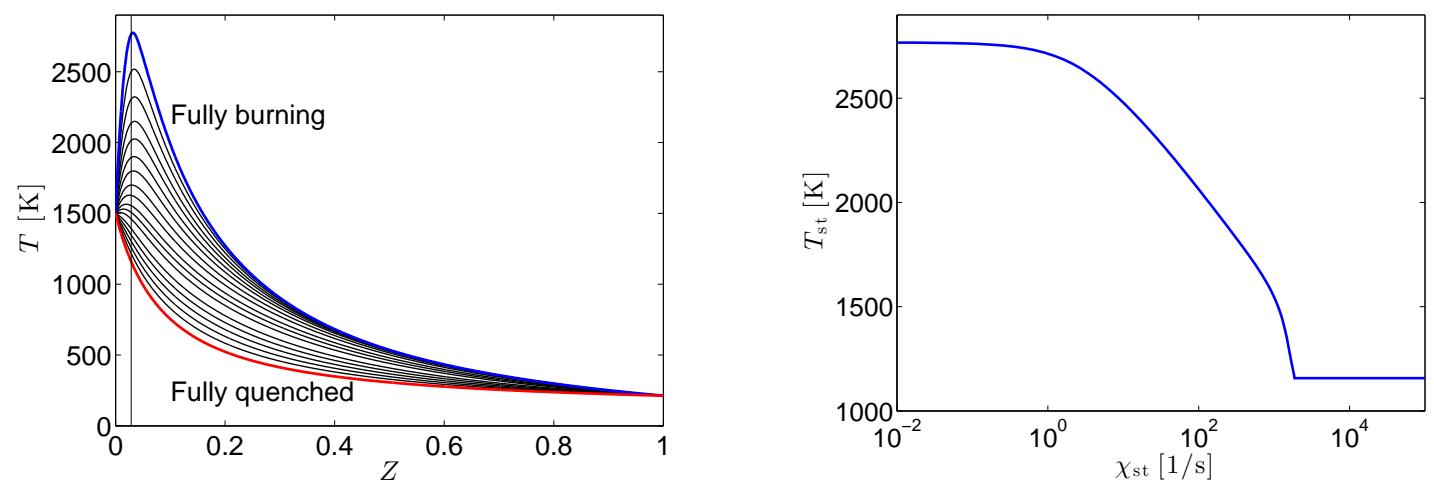

Figure 3: Flamelets used to model the $\mathrm{H}_{2}$-air chemistry, computed at a reference pressure of 1.5 bar. Left: Temperature as a function of the mixture fraction $Z$, with stoichiometric mixture marked by the vertical line. The flamelets in the limits of zero and infinite stretch are shown in thick lines, with approximately every fifth intermediate flamelet shown in thin lines. Right: Stoichiometric temperature as a function of the flame stretch $\chi_{\text {st }}$. Note the absence of an "S-shape", which is due to the high oxidizer temperature.

peratures at the fuel and oxidizer boundaries $\left(T_{\mathrm{tab}, Z=1}=210 \mathrm{~K}\right.$ and $T_{\mathrm{tab}, Z=0}=1500 \mathrm{~K}$, respectively). These conditions are representative of the conditions in the HyShot II combustor. The $\mathrm{H}_{2}$-air mechanism (9 species, 20 reactions) by Hong et al. [36] is used. Sample results of the pre-computed flamelets are shown in Fig. 3. The fully quenched (mixing only) stoichiometric temperature is $1160 \mathrm{~K}$. At such high temperatures, there is no hysteresis and thus no "S-curve", as shown in the figure.

The pre-computed flamelet solutions act as a tabulated equation-of-state in the LES code. The LES transport equations provide values of the density $\bar{\rho}$, the internal energy (sum of chemical and sensible) $\widetilde{e}$,

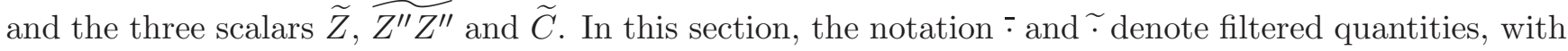
the latter including a density-weighting (in the results sections, these notations will be used for averages instead). To compute the remaining state variables $\bar{p}$ and $\widetilde{T}$, the approximate approach proposed by Pecnik et al. [21] is followed. The mass fractions $\widetilde{Y}_{k}$ for the 9 species are assumed to be functions of the three scalars only, thus independent of the pressure and temperature. Further assuming a linear variation of the ratio of specific heats $\gamma$ around the flamelet solution allows the filtered internal energy $\widetilde{e}$ to be approximately related to the filtered temperature $\widetilde{T}$ as

$$
\widetilde{e}(\widetilde{T}) \approx \widetilde{e}_{\mathrm{tab}}+\int_{\widetilde{T}_{\mathrm{tab}}}^{\widetilde{T}} \frac{R_{\mathrm{tab}}}{\gamma_{\mathrm{tab}}+\gamma_{\mathrm{tab}}^{\prime}\left(\Theta-\widetilde{T}_{\mathrm{tab}}\right)-1} d \Theta,
$$

where $\widetilde{T}_{\text {tab }}$ is the filtered flamelet temperature (using the $\beta$-PDF) and $R_{\text {tab }}$ is an effective gas constant for the filtered mixture composition in the flamelet table, defined as $\widetilde{R T} / \widetilde{T}$. Similarly, $\gamma_{\text {tab }}$ and $\gamma_{\text {tab }}^{\prime}$ are an effective filtered ratio-of-specific-heats and its derivative, defined from the derivative of $\widetilde{e}$ with respect 
to $\widetilde{T}$. Eqn. (1) can be inverted to

$$
\widetilde{T}=\widetilde{T}_{\mathrm{tab}}+\frac{\gamma_{\mathrm{tab}}-1}{\gamma_{\mathrm{tab}}^{\prime}}\left(\exp \left\{\frac{\gamma_{\mathrm{tab}}^{\prime}\left(\widetilde{e}-\widetilde{e}_{\mathrm{tab}}\right)}{R_{\mathrm{tab}}}\right\}-1\right),
$$

which allows for the temperature to be computed given $\widetilde{e}$ and the three scalars (which determine the tabulated values). The filtered pressure is then simply computed as $\bar{p}=\bar{\rho} R_{\mathrm{tab}} \widetilde{T}$. The viscosity and thermal conductivity are computed using one-parameter power-law expansions around the tabulated conditions as $\sim\left(\widetilde{T} / \widetilde{T}_{\mathrm{tab}}\right)^{a}$, where the exponent $a$ is computed for each quantity by perturbation around the flamelet solution and stored in the table.

Pecnik et al. [21] proposed that the first-order effect of flow-induced variations in pressure and enthalpy is to modify the source term of the progress-variable $\widetilde{C}=\widetilde{Y}_{\mathrm{H}_{2} \mathrm{O}}$, and that this can be modeled to leading order by introducing a pressure-scaling in the filtered source term $\dot{\omega}_{\widetilde{C}}=\overline{\dot{\omega}}_{\mathrm{H}_{2} \mathrm{O}}$ (i.e., the production-rate of water, units $\left.\left[\mathrm{kg} /\left(\mathrm{m}^{3} \mathrm{~s}\right)\right]\right)$. Saghafian [32] used a DNS database of a temporally evolving mixing layer for an a priori assessment, and found that the source term $\dot{\omega}_{\widetilde{C}}$ scales quadratically with pressure to a very good approximation. Inspired by these studies, the source term is modeled in this work as

$$
\dot{\omega}_{\widetilde{C}}=\left(\frac{\bar{p}}{p_{\text {tab }}}\right)\left(\frac{\bar{\rho}}{\bar{\rho}_{\text {tab }}}\right) \dot{\omega}_{\widetilde{C}, \text { tab }} .
$$

The scaling with density implies that the specific production-rate $\dot{\omega}_{\widetilde{C}, \text { tab }} / \bar{\rho}_{\text {tab }}$ (units $[1 / \mathrm{s}]$ ) is taken from the flamelet table, and the extra pressure-factor then leads to an approximate quadratic pressure-dependency.

We end by pointing out that the modeling choices used here, particularly the assumption that minor species concentrations do not depend directly on pressure and enthalpy changes, is reflective of the focus on major flow behavior in the present study. A more accurate approach might be required if minor species are of interest, or perhaps in the blow-out limit; in that case, one could introduce additional dimensions in the flamelet table accounting for pressure and enthalpy variations. Note also that Saghafian [32, 37] proposed and tested a correction factor for temperature variations in the progress-variable source term. This was found to have an effect only very near the walls in the present work (the HyShot II geometry with wall-modeled LES), and was thus not included in the model; for other problems, including this could be of value.

\subsection{Wall-model and grids}

The friction Reynolds number $R e_{\tau}$ of the boundary layers in the HyShot II isolator and combustor varies from 1500 to 4000; with reasonable resolution of the viscous length scale this implies that a grid of order $10^{11}$ points would be needed with traditional (wall-resolved) LES. Since this is completely impractical, one might wonder whether it is possible to obtain reasonable predictions of the flow by simply ignoring the viscous near-wall region, i.e., by knowingly underresolving this region. After all, if the pressure rise is due to the heat release, which occurs in the mixing layer between the fuel jet and the surrounding air, then perhaps the near-wall processes are not important? 


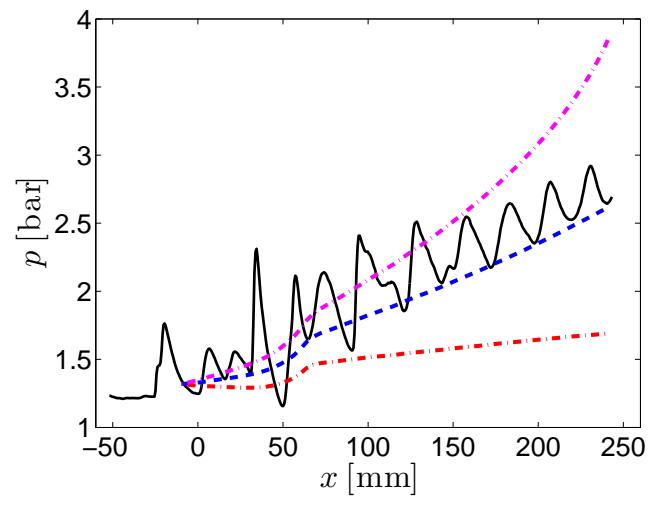

Figure 4: Rayleigh-Fanno analysis of pressure increase in the HyShot II combustor. Pressure on the lower wall from RANS (solid) compared with the Rayleigh-Fanno result with integrated heat release, friction and wall heat losses (dashed). Also shown are the Rayleigh-Fanno results without the friction component (dash-dotted, lowest pressure-rise) and without the wall heat losses (dash-dotted, highest pressure-rise).

To answer this question we utilize a simple Rayleigh-Fanno analysis, i.e., inviscid 1D flow of a perfect gas with heat addition and friction. Figure 4 shows the pressure on the lower wall from 3D reacting RANS [21] and the equivalent Rayleigh-Fanno result with the heat release, heat losses and friction taken from the RANS. The latter obviously does not include non-uniform 3D effects or shock waves, but nevertheless gives a similar pressure rise (by design). Within the Rayleigh-Fanno framework it is then straightforward to compute the pressure rises without the effects of wall friction or wall heat losses; these are also shown in the figure. The effects of these phenomena on the pressure rise are dramatic. The 'true' pressure rise is 1.3 bar; neglecting friction causes a -0.9 bar difference whereas neglecting wall heat losses causes a +1.3 bar difference. The conclusion is that near-wall processes are of paramount importance in this scramjet flow, and thus they must be accurately predicted by the LES. This is accomplished by using a wall-model.

The wall-model used here is taken from the work of Kawai and Larsson [38], who both extended the previously existing equilibrium-stress approach to compressible flows with wall heat transfer and proposed a novel idea for how to minimize the error in the predicted skin friction. Following this, the wall-model is solved in the lowest $10 \%$ of the boundary layer (based on $\delta_{99}$ ).

The three scalar equations for $\widetilde{Z}, \widetilde{Z^{\prime \prime} Z^{\prime \prime}}$ and $\widetilde{C}$ also need closure in the absence of resolving the nearwall region in the LES. For all three equations a zero-flux condition is used. This clearly makes sense for $\widetilde{Z}$ and $\widetilde{C}$. The transport equation for the mixture fraction variance $\widetilde{Z^{\prime \prime} Z^{\prime \prime}}$ has a production term that is not captured in the LES. The zero-flux condition is an approximation for this quantity.

The wall-model has been validated on supersonic boundary layers as well as on the shock/boundary layer interaction in an almost square duct studied experimentally by Helmer, Campo and Eaton [39, 40]. This problem validates the capability of the wall-modeled LES to capture stress-induced secondary corner flows and, most importantly, shock/boundary layer interaction; results from this validation exercise are 
described in Bermejo-Moreno et al. [41], and will not be repeated here.

With the use of the wall-model, the grid resolution is determined solely by the boundary layer thickness (i.e., not by the viscous length scale). The grids are mainly structured, with O-grids in and around the fuel injector. Three different grids are used to estimate the degree of grid sensitivity in the results, with total cell-counts of 100M (fine), 43M (medium), and 14M (coarse). These are all finer than the grids used by Chapuis et al. [23], which used 51M cells for the full width of the combustor (equivalent to about 13M cells for a single injector). The spanwise grid spacing throughout the domain, and the streamwise grid spacing in the region around the fuel injector, is $0.075,0.10$ and $0.15 \mathrm{~mm}$ for the three grids, respectively. The streamwise grid spacing is stretched towards the inlet and the region where the combustion occurs (twice and three times larger grid-spacing compared to near the injector, respectively). The wall-normal grid spacing is equal to the spanwise one in the core of the combustor, and smoothly refined towards the walls (three times finer grid).

The boundary layers are approximately $1 \mathrm{~mm}$ thick throughout most of the domain (slightly thinner near the inlet, slightly thicker in the combustor region); thus the grid resolution is somewhat coarse in the boundary layers compared to the criteria given by Kawai and Larsson [38]. Note that the wall-model is not applied in the injector itself, since the flow there is laminar due to the strong contraction.

\section{Validation at started conditions}

The first experimental campaign in the HEG shock tunnel at DLR in Germany using the HyShot II configuration considered two different operating conditions, replicating the flight conditions at altitudes of 27 and $33 \mathrm{~km}$, respectively [9]. The model instrumentation was restricted to wall pressure and heat flux gauges mounted on the injector-side combustor wall. In order to improve the wall instrumentation (heat flux and pressure gauges on the intake, the injector-side and cowl-side combustor walls, and the exhaust surfaces) and to allow optical access to the combustor, a new wind tunnel model was subsequently designed and manufactured. The first test campaign utilizing this new model focused again on the 27 and $33 \mathrm{~km}$ altitude HEG conditions [10, 11]. All simulations in this paper correspond to the $27 \mathrm{~km}$ case, or more specifically to the HEG conditions aimed at replicating the $27 \mathrm{~km}$ case. For this case, the majority of the HEG runs were performed using an angle of attack of $3.6^{\circ}$. The fuel/air equivalence ratio was varied between $E R=0$ and 0.61 , resulting in steady and unsteady combustor flows.

A subset of 9 experimental runs with reacting flow near the nominal operating condition are available from the campaign by Hannemann et al. [10, 11]; these have nominal equivalence ratios (ERs) from 0.27 to 0.35 due to run-to-run variations. The run with the highest ER (run \#810) is chosen for the validation study, since this can be expected to be the most challenging (more heat release, stronger fuel jet, etc). To estimate the uncertainty in the measurements, the variation in the measured values for the 3 runs with the highest ERs (having nominal ERs of 0.33, 0.34, 0.35) is used as a rough estimate of both the run-to-run variability and the uncertainty in the experimentally estimated value of the ER; the data from these additional runs are also shown in the figures. The fuel stagnation pressure $p_{0, \text { fuel }}$ is 5.73 bar for run \#810. 

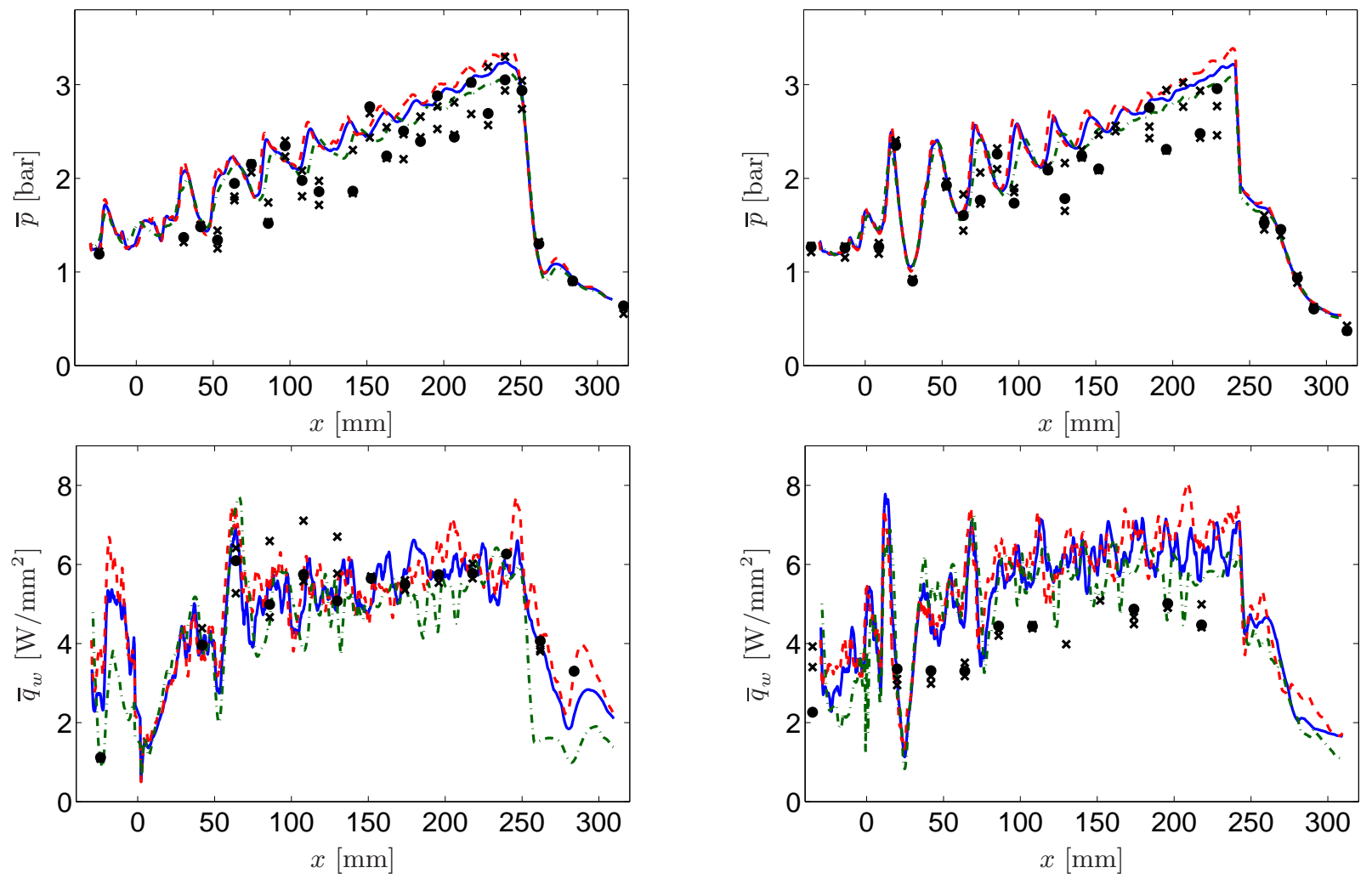

Figure 5: Mean wall pressure and heat flux in HyShot II from LES (lines) for run \#810 with ER=0.35. Compared with experiments $[10,11]$ for the same run \#810 (circles) as well as runs \#804 and \#809 (ERs=0.34 and 0.33; both marked with crosses) to show the run-to-run variation. LES on fine mesh (100M cells, dashed), medium mesh (43M cells, solid) and coarse mesh (14M cells, dash-dotted). Top row: wall pressure along a line halfway between the injectors. Bottom row: wall heat flux along a line through the injector. Left column: Lower wall. Right column: Upper wall.

The quantitative experimental data available are measurements of pressure and heat flux along lines on the lower and upper walls in the combustor. Pressure is measured between injectors, while heat fluxes are measured along lines intersecting the injectors. The comparison between this data and the LES results is shown in Fig. 5.

In this and all following sections, the notation ${ }^{\sim}$ and $\sim$ denotes time-averaged quantities, with densityweighting for the latter.

We first note that the LES results for the mean pressure on the three grids are relatively close to each other, indicating that the results are close to (but not quite) grid-converged for this quantity. The experimental results suggest large variations between subsequent pressure taps; the LES, on all three grids, has much lower variation in the mean pressure. The reason for this discrepancy is not clear: the obvious explanation would be that the (numerically captured) shocks in the LES are smeared, but if this were the case there should be a larger difference between the results from the three grids. 


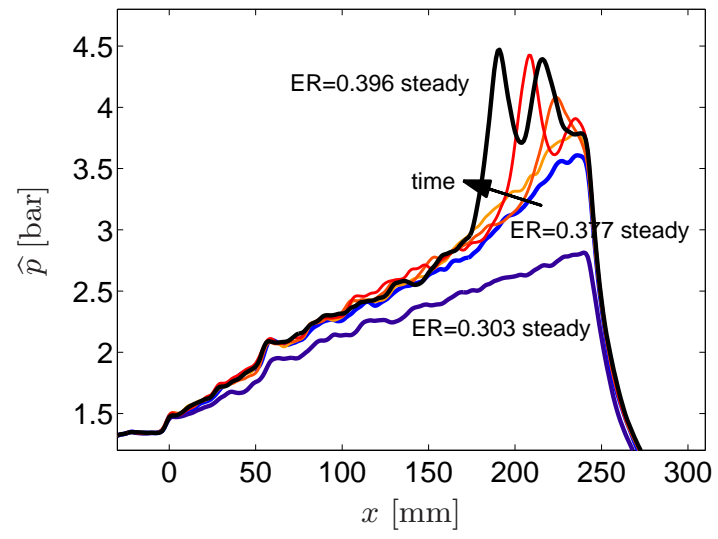

Figure 6: Effect of increasing ER on the cross-sectionally averaged pressure $\widehat{p}$. Steady state at the nominal operating point $\mathrm{ER}=0.303$ (lowest line). Steady state at $\mathrm{ER}=0.377$ is qualitatively similar (second lowest line). Increasing to $\mathrm{ER}=0.396$ creates a "combustor shock-train", where solutions are shown after $0.8 \mathrm{~ms}, 1.2 \mathrm{~ms}, 2.0 \mathrm{~ms}$ and the long-term steady solution after about $9 \mathrm{~ms}$ (most upstream line).

The computed mean pressure profiles fall along the upper bound of the experimental data for the same reported $E R=0.35$. The experiment has about $8 \%$ uncertainty in the estimated ER [13], and thus the cases at reported ERs of 0.34 and 0.33 (lower by $3 \%$ and $6 \%$, respectively) actually fall within the margin-of-error in terms of the ER. Since these ERs are nominally lower, the corresponding results should be seen as an approximate lower bound on the measurements. In the absence of cases with nominally higher ERs, it is reasonable to presume a similar margin-of-error towards larger values. With this, the computed pressure profiles all fall well within the experimental uncertainty.

\section{Increasing the fuel/air equivalence ratio (ER)}

The main focus of this study is on investigating the changes in the flow when the fuel/air equivalence ratio (ER) is increased. The incoming air flow is held constant at the average shock tunnel conditions for the remainder of the paper; thus the ER is directly controlled by the total pressure in the fuel feed $p_{0, \text { fuel }}$. For the fixed incoming air flow, the ER is approximately 0.0609 times the value of $p_{0 \text {,fuel }}$ measured in bar.

For ER $\lesssim 0.38$, increasing the ER does not lead to any qualitative changes in the flow, only to a slightly larger pressure-rise within the combustor and a larger thrust. Increasing the ER beyond about 0.38 , however, results in a qualitative change in the flow. This is illustrated in Fig. 6 , which shows how the cross-sectionally averaged pressure $\widehat{p}$ is affected by a sudden change in ER. This particular simulation was started from an instantaneous realization of the flow from the statistically steady state at $\mathrm{ER}=0.377$, with "nominal" flow in the combustor (i.e., approximately linear pressure rise). The fuel pressure $p_{0 \text {,fuel was }}$ then instantly raised from 6.2 bar to 6.5 bar, corresponding to $E R=0.396$. For the first $0.8 \mathrm{~ms}$, the only visible change is a larger pressure in the second half of the combustor; however, the flow is qualitatively the same. After $1.2 \mathrm{~ms}$, there has been a fundamental change in the flow topology, with the development 


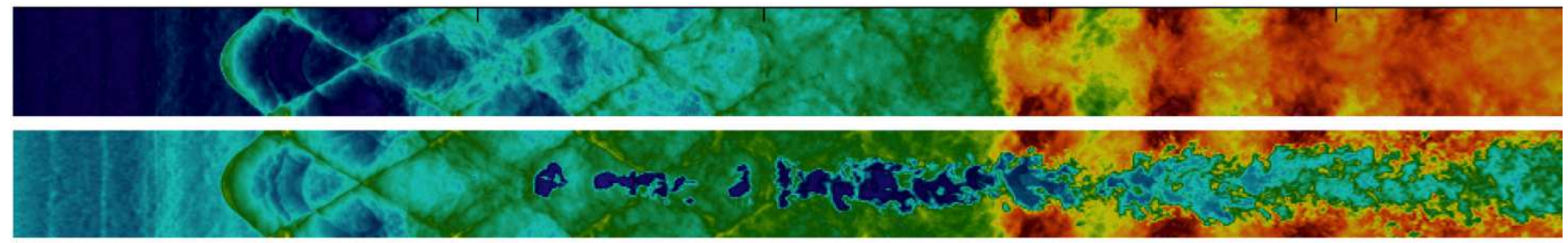

Figure 7: Contours in a horizontal plane at $y=7 \mathrm{~mm}$ (i.e., $2.8 \mathrm{~mm}$ from the upper wall), from $x=-30 \mathrm{~mm}$ to $x=242 \mathrm{~mm}$ (the combustor exit), for $\mathrm{ER}=0.414$. Colored from low (blue) to high (red) values. Top: pressure (0.6 to 6.0 bar). Bottom: density $\left(0.1\right.$ to $\left.1.1 \mathrm{~kg} / \mathrm{m}^{3}\right)$.

of a stronger shock towards the end of the combustor. For later times, this shock travels upstream, becoming the leading shock in a shock-train, and finds a seemingly stable position in the combustor. This shock-train is similar to the shock-trains that form in the isolator of scramjets in dual-mode operation, but differs in that here it forms in the combustor, concurrent with the mixing and heat release. For this reason, and to distinguish it from more common isolator shock-trains, we refer to it as a "combustor shock-train" in this paper.

The flow is partially visualized in Fig. 7. The combustor shock-train begins with the sharp rise in pressure and density towards the end of the combustor. Note the co-existence (or co-location) of the combustor shock-train and the fuel jet. The term "leading shock" is used to denote the beginning of this combustor shock-train. The location of the leading shock is denoted by $x_{s}$, and is defined as the point where the cross-sectionally averaged Mach number $\widehat{M}$ first decreases below 1.1. This location is not very sensitive to the exact threshold, nor to which quantity is used to define it, with variations limited to within 2-3 $\mathrm{mm}$.

We note that the appearance of a combustor shock-train for ER $\gtrsim 0.40$ here is consistent with the results of Chapuis et al. [23], who noticed a qualitative difference in the results between the flight at 33 $\mathrm{km}$ altitude and the shock tunnel experiments designed to approximate that flight condition. The ER was about 0.38 in the flight but around 0.43 in the experiments, and the pressure profile in the latter case appears similar to the present ones with a combustor shock-train.

The range of ERs that result in a combustor shock-train is the focus of the remainder of the paper. Comparisons will be made with a series of shock-tunnel experiments, described more fully in the companion paper [15] to the present one. It should be noted that, although the present LES investigation into this regime was launched in parallel with the experimental investigation in the HEG shock tunnel at DLR, no further communication between the respective parties took place before the simulation details were finalized and most of the results were obtained. The simulations were thus truly blind predictions.

\subsection{Long-time integration and validation}

The first step is to verify that the simulations reach a truly steady state, and that the combustor shock-train does not continue moving upstream eventually causing inlet unstart. Fig. 8 shows an $x-t$ contour of the cross-sectionally average pressure $\widehat{p}$ for $\mathrm{ER}=0.414$. The highest pressures form "ridges" in 


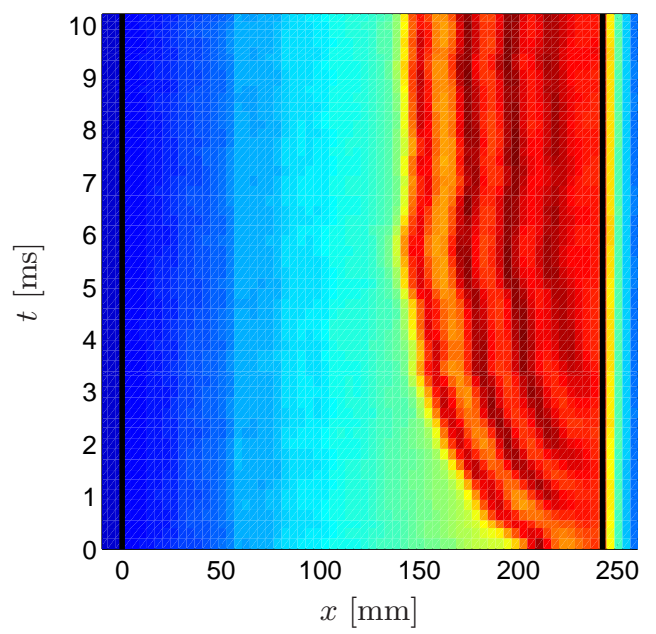

Figure 8: Cross-sectionally averaged pressure $\widehat{p}$ from the medium mesh at $\mathrm{ER}=0.414$, plotted versus streamwise location and time from $1.1 \mathrm{bar}$ (white/blue) to $4.6 \mathrm{bar}$ (black/red). The injector is located at $x=0$ and the nozzle begins at $x=242.5$ mm (marked by a thick line).

the figures; the first (most upstream) ridge is the leading shock of the combustor shock-train.

This case was integrated for $10 \mathrm{~ms}$, about twice the test time in the experiments at DLR. The solution clearly reaches a steady state after about $5 \mathrm{~ms}$, and then stays in place for the remaining $5 \mathrm{~ms}$ of the simulation. This length of time corresponds to approximately 25 flow-through times. We conclude that the flow is in a statistically steady state, at least on the time scales investigated here.

The evolution of the leading shock location $x_{s}$ is shown in Fig. 9 for several different ERs. The speed of the leading shock $u_{s}=d x_{s} / d t$ is also shown in the figure. Each case was started from the next lower ER. The combustor shock-train appears to reach a steady position within the combustor for ERs up to 0.450 , as evidenced by both the $x_{s}$ and the (more sensitive) $u_{s}$ profiles. At $\mathrm{ER}=0.469$, the solution has not been integrated for sufficiently long to say. At $\mathrm{ER}=0.524$, the combustor shock-train moves upstream until the leading shock reaches about $x_{s} \approx 30 \mathrm{~mm}$, at which point the shock system merges with the fuel jet bow shock (at $t \approx 3 \mathrm{~ms}$ ). This behavior is similar to that seen in a RANS simulation of the same configuration at $\mathrm{ER}=0.553$ [13]. At $\mathrm{ER}=0.597$, the shock-train continues to move farther upstream into the isolator, until it reaches the inlet to the LES domain and the simulation is halted.

The simulations thus show three distinctly different flow regimes: nominal, with supersonic combustion throughout, for $\mathrm{ER} \lesssim 0.38$; stable and steady flow with a combustor shock-train for $0.38 \lesssim \mathrm{ER} \lesssim 0.5-0.6$; and either classic dual-mode flow with an isolator shock-train or fully unstarted flow for ER $\gtrsim 0.6$.

The final location of the combustor shock-train $x_{s, \text { final }}$ is shown in Fig. 10 and compared to the results from the experiments $[12,15]$. For both the LES and the experiments, the uncertainty due to variations in position over the finite time extent is estimated and shown in the figure. The agreement is rather remarkable for ER $\lesssim 0.45$, but with differences for larger ERs. In fact, the earlier experiments of Laurence et al. [13] indicated that the combustor shock-train may be stable for even larger ERs (up to 

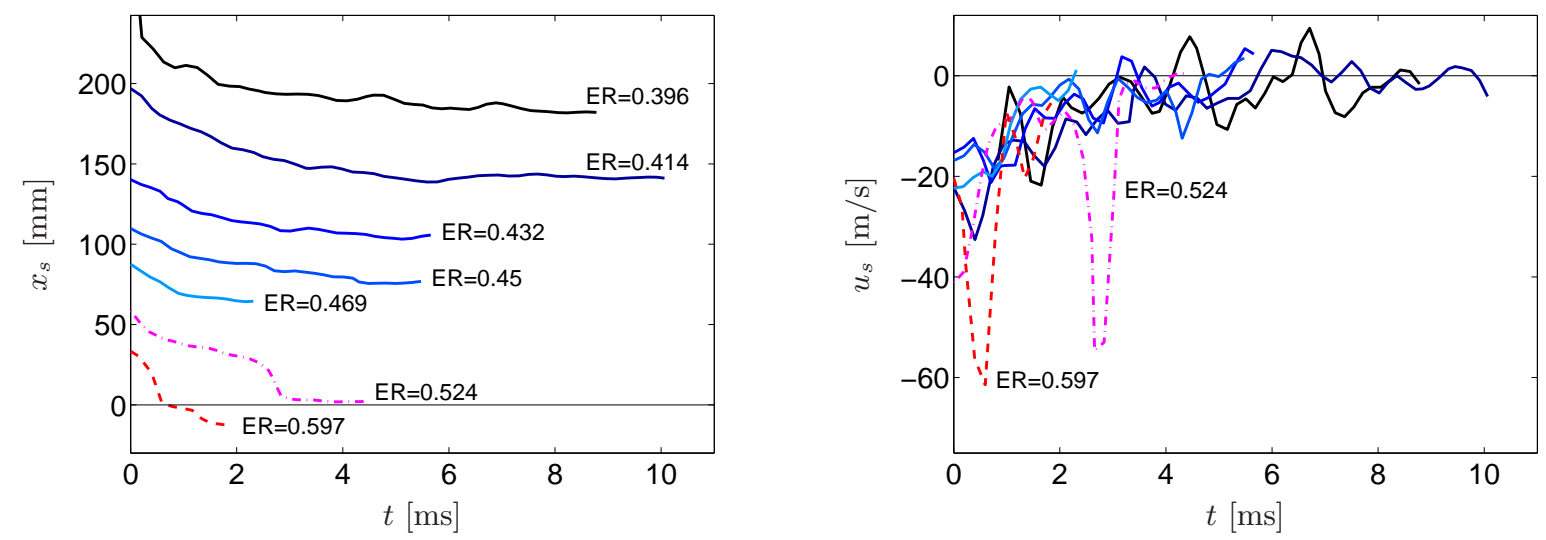

Figure 9: Evolution in time of the location of the leading shock in the combustor shock-train $x_{s}$, defined as the location where the cross-sectionally averaged Mach number $\widehat{M}$ first dips below 1.1. Left: Leading edge location $x_{s}$ for a collection of ERs. Note that the fuel injector is located at $x=0$. Right: Velocity $u_{s}=d x_{s} / d t$ for the same ERs, filtered to remove noise due to the numerical differentiation.

0.7) than those considered here, at least within the limitation of the short test time.

A Schlieren image from the experiment at $\mathrm{ER}=0.414$ is shown in Fig. 11. For comparison, the densityderivative in the streamwise direction from the LES is integrated in the spanwise direction to emulate the Schlieren. The same flow structures are seen, although they are more smeared in the experiment; this is partly due to the experimental image being from the full combustor with four injectors, whereas the LES computes only a single one. The leading shock in the combustor shock-train is indicated in the experimental image, but is hard to distinguish in the LES result.

A more detailed validation is done by comparing the steady state pressure profiles along the lower wall at four different ERs between the LES and the experiments. We note that the LES computations were performed before the experiments were concluded, which led to slight differences in the air inflow conditions. Specifically, the LES computations use the nominal shock tunnel conditions from the earlier experimental HyShot II campaign [10, 11]. As explained by Laurence et al. $[12,15]$, however, the shock tunnel conditions in the most recent experimental study differ slightly, by about $5 \%$ in the stagnation pressure. This discrepancy is partially accounted for here by scaling the experimental pressure data by the ratio of the stagnation pressures.

The experimental pressure profiles averaged during the quasi-steady test time are compared with profiles averaged during the steady part of the LES runs in Fig. 12. The first thing to notice is the collapse of the pressure profiles up to the leading shock in the combustor shock-train among both the experimental runs and the simulation results. This is due to the low momentum flux ratio at these ERs, coupled with the fact that the combustion during the initial phase is insensitive to the amount of fuel injected. There is a qualitative agreement between the computed and experimental results at each ER, although the simulations consistently show higher pressure and a leading shock (for the two highest ERs) that is slightly more upstream. Some caution is needed, however. First, as Fig. 10 shows, the nominal ER values 


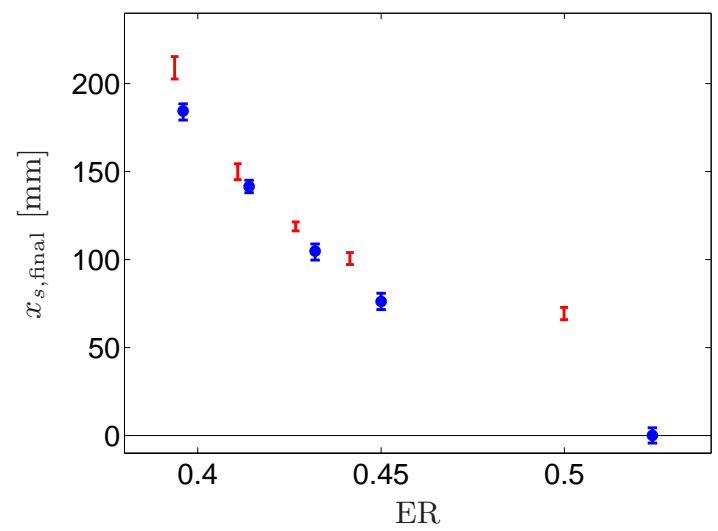

Figure 10: Final leading shock location $x_{s, \text { final }}$ for different ERs. Comparison of results from LES (blue, with circles) with those from the experiment (red [15]). To facilitate direct comparison, the $x_{s, \text { final }}$ is extracted here (in this figure) from the spanwise-averaged density variation near the upper wall, analogously to how it was extracted from the experimental Schlieren images. The error estimates are primarily due to the finite time extent (thus largest for the experiment and the LES cases

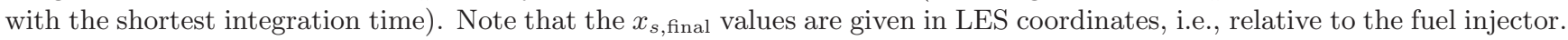

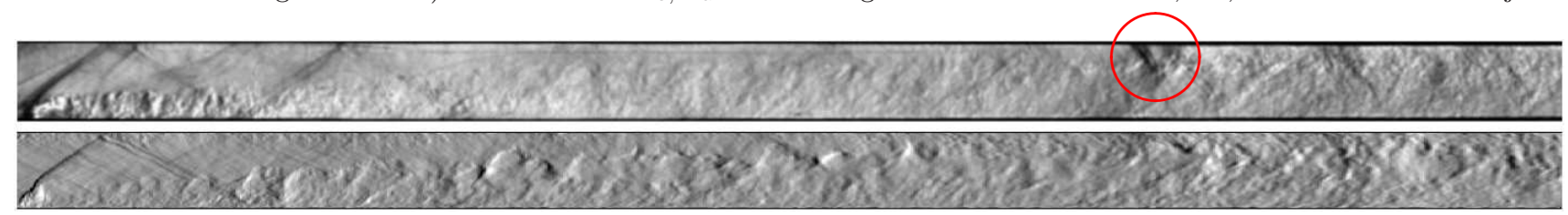

Figure 11: Schlieren images at $\mathrm{ER}=0.414$. Top: Experiment with vertical knife-edge, with a circle showing the leading shock in the combustor shock-train. Bottom: LES, showing $\partial \rho / \partial x$ integrated over the spanwise direction.

do not match exactly. Secondly, the experimental ER values have about $8 \%$ uncertainty [13]. Therefore, Fig. 12 shows that the predicted LES pressure profiles fall well within the experimental uncertainty. We also note that the strength of the trailing shock structures decreases progressively as the ER is increased in both the LES and the experiments.

This concludes the validation phase of the study into higher ERs. To within the uncertainties, and to within the scope of the present study (main flow features, not minor species), the LES can be considered to be in rather good agreement with the experimental data. This is particularly true given the fact that these were blind predictions.

It is also worth pointing out that the LES and the experiments arrive at similar end states from very different initial transients during each run. In the LES, the air flow is held constant while the fuel mass flux is increased (compared to the initial condition); in the experiments, the fuel flow is essentially constant during each test, and the flow goes through a start-up period during which the air flow stabilizes. The fact that both LES and experiments arrive at similar end states suggests that there is no hysteresis in this problem. 

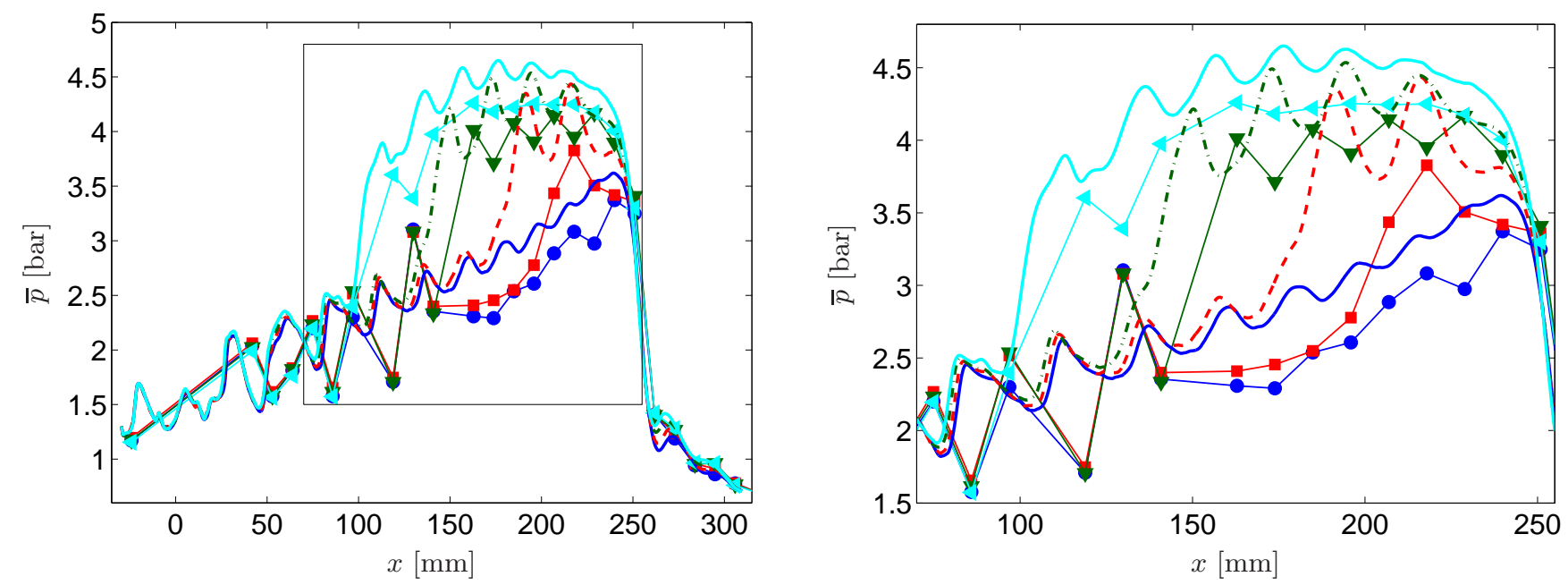

Figure 12: Mean wall pressure on the lower wall halfway between the injectors from LES (lines) and experiments by Laurence et al. $[12,15]$ (symbols). LES results at ER of 0.377 (solid), 0.396 (dashed), 0.414 (dash-dotted) and 0.432 (solid again). Experimental results at ER of 0.38 (circles), 0.39 (squares), 0.41 (downward triangles) and 0.43 (leftward triangles); averaged over the quasi-steady test time. Left: full view. Right: zoomed view, in the window shown in the left figure.

\subsection{Analysis and visualization}

The focus of this section is to investigate and characterize the statistically steady flow in the regime with a combustor shock-train.

The basic effect of increasing the equivalence ratio (ER) is shown in Fig. 13. which shows quantities averaged in time and over the combustor cross-section (or across the upper and lower walls in the case of surface quantities). Note that simple cross-sectional averages are used, without any stream-thrust weighting or similar. The cross-sectionally averaged Mach number is close to 1 in the combustor shocktrain, and about $40-60 \%$ of the cross-section is occupied by subsonic flow for those cases. For ER=0.414, the shock-train is not strong enough to drive the average flow to subsonic conditions, though for ER $=0.450$ $\widehat{M}$ becomes principally subsonic a short distance downstream of the leading shock. Increasing the ER to 0.524 pushes the combustor shock-train up to the fuel injector, where it merges with the fuel jet bow shock. This case produces up to $70 \%$ subsonic flow, yet it appears to be stable.

In the plot of the averaged source term, $\widehat{\dot{\omega}}_{\mathrm{H}_{2} \mathrm{O}}$, (upper right) we see that the presence of the shock-train substantially increases $\mathrm{H}_{2} \mathrm{O}$ production (and by proxy, the rate of heat release) in the region immediately downstream of the leading shock. This is consistent with the intensified $\mathrm{OH}^{*}$ intensity seen associated with the shock-train in the experimental chemiluminescence images of Laurence et al. [15]. The average $\widehat{\dot{\omega}}_{\mathrm{H}_{2} \mathrm{O}}$ tails off significantly downstream.

In the lower left plot we see that the shock-train causes the wall friction to decrease by approximately $10 \%$ in comparison to the $\mathrm{ER}=0.377$ case. Much more significant, however, is the enhanced heat loss at the combustor walls (lower right), which can increase by $50 \%$ or more. Both these effects - decreased 

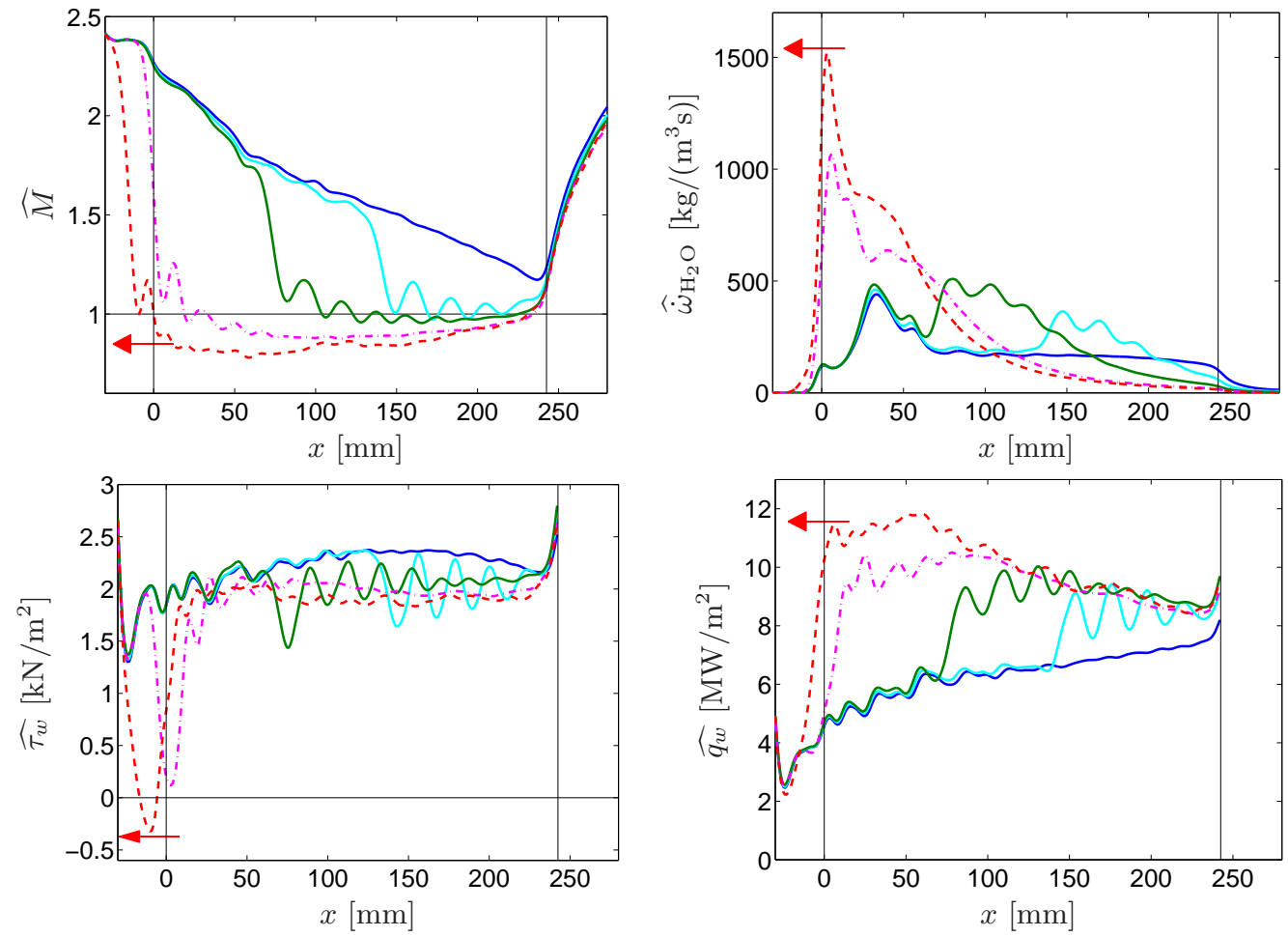

Figure 13: Time- and cross-sectionally averaged results during steady state at different ERs. In every plot, the different cases are, in order, $\mathrm{ER}=0.377$ (nominal, fully started flow; blue solid line), $\mathrm{ER}=0.414$ (with combustor shock-train starting at $x_{s} \approx 140 \mathrm{~mm}$; light blue solid), $\mathrm{ER}=0.450$ (combustor shock-train starting at $x_{s} \approx 75 \mathrm{~mm}$; green solid), ER=0.524 (combustor shock-train merged with the fuel injector bow shock; pink dash-dotted), and ER=0.597 (combustor shock-train starting upstream of the injector, and moving upstream as indicated by the arrow; red dashed). Note that the ER=0.597 case does not reach steady state, and the simulation was halted due to the combustor shock-train reaching the LES inflow.

friction and increased heat loss - will tend to drive the combustor flow away from choking conditions.

The flow is visualized in Fig. 14 in a plane through the fuel injector. In this and most subsequent figures, three representative cases are shown: $\mathrm{ER}=0.377$ is the highest $\mathrm{ER}$ that still displays a nominal operating pattern, $\mathrm{ER}=0.414$ with a combustor shock-train starting at $x_{s} \approx 140 \mathrm{~mm}$, and $\mathrm{ER}=0.450$ with a combustor shock-train starting at $x_{s} \approx 75 \mathrm{~mm}$. The chemical reactions are particularly vigorous immediate behind the leading shock in the shock-train, as seen to some degree in the instantaneous temperature and $\mathrm{OH}$ mass fraction visualizations and more clearly in the cross-sectionally averaged results in Fig. 13.

The boundary layers are fully attached, even instantaneously, at $\mathrm{ER}=0.377$, but a separation bubble appears near the leading shock in the combustor shock-train. This bubble is instantaneously rather large in the vertical direction, but of limited extent in the spanwise direction (not shown). When present, it always appears on the lower wall near the centerline, in the lower-velocity and lower-density wake of the 
fuel injection.

The $\mathrm{H}_{2} \mathrm{O}$ source term in Fig. 14 shows quite clearly how the flame is anchored upstream of the fuel injector, in the small recirculation region caused by the bow shock around the fuel jet. The flamelet-based combustion model then predicts a partially quenched flame up to about $x \approx 30 \mathrm{~mm}$, as evidenced by the low temperature and $\mathrm{H}_{2} \mathrm{O}$ mass fraction in Fig. 14. In the remainder of the combustor, the flamelet-based combustion model predicts fast chemistry behavior. As a representative example, the chemical state at $x=115 \mathrm{~mm}$ for $\mathrm{ER}=0.377$ is shown in Fig. 15. The mass fraction of water is essentially that of an equilibrium flamelet. The results by Karl et al. $[16,17,18,19]$ using a finite rate chemistry RANS model support the notion that the chemistry truly is close to equilibrium in most of the combustor. Therefore, the most salient message of Fig. 15 is arguably the strong influence of the wall-cooling. The temperature range among the fully burnt gases is about $500 \mathrm{~K}$ to $2600 \mathrm{~K}$. Most of the strong wall-cooling affects the burnt gases.

We next consider the $\mathrm{H}_{2} \mathrm{O}$ production rate in more detail, as a surrogate of heat release. The instantaneous mass fraction of the reaction product $\mathrm{H}_{2} \mathrm{O}$ is shown in slices at constant $x$ in Fig. 16. The stoichiometric line $Z=0.028$ is shown in each figure, and it is clear that the turbulent combustion model predicts fast chemistry behavior. The burnt gases inside the stoichiometric line are close to equilibrium, and there is a rather sharp reaction front around the stoichiometric line.

The sonic line is also shown in the figures and displays an interesting behavior. For the nominal flow pattern at $\mathrm{ER}=0.377$, the sonic line is clearly separated from the stoichiometric line at both $x$ locations. This is also true for $\mathrm{ER}=0.414$ at $x=115 \mathrm{~mm}$, which is ahead of the beginning of the combustor shocktrain. In the remaining plots in Fig. 16, however, the stoichiometric and sonic lines almost coincide. These figures are all taken from cases/locations within the combustor shock-train. While one might expect the stoichiometric and sonic lines to cross at some point (some $x$ for some ER), the fact that they nearly coincide for an extended range (of $x$ and ER) is surprising. This is also seen in Fig. 14, which shows how the sonic and stoichiometric lines stay very close together from the start of the combustor shock-train $\left(x_{s} \approx 75\right.$ and $140 \mathrm{~mm}$ at $\mathrm{ER}=0.414$ and 0.450 , respectively) and for about $40 \mathrm{~mm}$ downstream. The fact that this occurs instantaneously, and not just on average, suggests that this is not a coincidence.

A three-dimensional visualization of this is shown in Fig. 17, which shows how the sonic and stoichiometric surfaces never really cross each other at nominal operation $(\mathrm{ER}=0.377)$. However, they do cross at the beginning of the combustor shock-train, and it is only after about $40 \mathrm{~mm}$ that the sonic surface truly moves outside of the stoichiometric surface.

To see this effect in a more quantitative manner, the source term $\dot{\omega}_{\mathrm{H}_{2} \mathrm{O}}$ is conditioned on the local Mach number and shown in Fig. 18. In areas of nominal flow (all $x$ for $\mathrm{ER}=0.377, x \lesssim 140 \mathrm{~mm}$ for $\mathrm{ER}=0.414$ ), the source term is large predominantly in supersonic flow. For locations within the combustor shocktrain, however, the source term either straddles the sonic line or is centered immediately below it. The progression from $x=115 \mathrm{~mm}$ to $x=215 \mathrm{~mm}$ for $\mathrm{ER}=0.377$ is smooth, with the curve shifting steadily towards lower Mach numbers. At $\mathrm{ER}=0.414$, however, there is a more drastic shift around $x \approx 140 \mathrm{~mm}$, which is the beginning of the combustor shock-train.

In the experimental counterpart to the present study, we suggest that one reason that the shock-train can remain in the constant-area combustor (apparently contradicting a simple Rayleigh-flow analysis) is 
that it may lead to the heat release becoming more uniformly distributed over the combustor cross section. This would alleviate the "local" thermal choking that was found to be responsible for the initial formation of the shock-train $[20,13]$. In Fig. 19, the fraction of the cross-sectional area over which $95 \%$ of the $\mathrm{H}_{2} \mathrm{O}$ production occurs (normalized at each $x$ ) is shown. This metric is a measure of the compactness of the heat release region. Traveling downstream, there is a steady spreading of the heat release region for all cases, consistent with a spreading of the fuel jet and mixing region. Note also from Fig. 13 that the heat release (as represented by $\widehat{\dot{\omega}}_{\mathrm{H}_{2} \mathrm{O}}$ ) is significant in the regions of increased spread. At the beginning of the combustor shock-trains, the spreading-rate (slope) increases by a factor of 2 for $\mathrm{ER}=0.414$ and slightly more for $\mathrm{ER}=0.450$. This is comparable to the increase in cross-sectionally averaged $\mathrm{H}_{2} \mathrm{O}$ production immediately behind the leading shock in Fig. 13 for these cases. In other words, upon entering the combustor shock-train, the rates of heat release and growth in area over which it occurs both increase by similar factors. This confirms that the explanation for the stability of the combustor shock-train in terms of its alleviation of the localized thermal choking downstream is at least plausible. We will return to this point shortly.

\subsection{Effect on combustor performance}

In part I of this study [15] we noted that the combustor shock-train may have the potential to improve combustor performance, as it appears to enhance mixing and combustion efficiency. Counting against this is the increased heat loss at the walls and the associated stagnation pressure losses, though the latter will be less severe than for a conventional isolator shock-train because of the reduced upstream Mach number. In this subsection we attempt to quantify these influences as they affect the overall combustor performance.

As shown in Fig. 20, the combustion efficiency increases from around $85 \%$ for a shock-train-free flow to over $91 \%$ for $\mathrm{ER} \gtrsim 0.45$. Here the combustion efficiency is based on the formation of $\mathrm{H}_{2} \mathrm{O}$; it would be higher still if based on the consumption of $\mathrm{H}_{2}$ instead. With fast chemistry, the combustion efficiency is mixing-limited. Therefore, the increase in combustion efficiency is strongly related to change of the stoichiometric surface shown in Fig. 17. At nominal operation $(\mathrm{ER} \lesssim 0.38)$ the stoichiometric surface does not close before the nozzle exit, implying that rich mixture leaves the combustor. At the higher ER, the stoichiometric surfaces closes in the nozzle, implying near-complete combustion.

The fast chemistry also implies that the peak in $\mathrm{H}_{2} \mathrm{O}$ production immediately behind the leading shock in the combustor shock-train (Fig. 13) must be caused by increased mixing behind the leading shock. This is consistent with the well-known amplification of turbulence kinetic energy and reduction in the turbulence length scale (both yielding faster mixing) that occurs in shock/turbulence interactions [42], as well as the deposition of additional baroclinic vorticity at the interfaces between the shocks and the mixing layer.

It is instructive to consider the balance of heat fluxes in the combustor when the equivalence ratio is increased. The present flamelet-based combustion model does not allow the heat release to be extracted exactly (due to the approximate scaling for variable pressure effects). However, in the fast chemistry limit, the production of $\mathrm{H}_{2} \mathrm{O}$ is closely correlated with the heat release, and thus we use the former as a good surrogate of the latter in this study. The global heat release is thus estimated from the $\mathrm{H}_{2} \mathrm{O}$ flux at 
the combustor exit together with its formation enthalpy, with the result shown in Fig. 21. The figure also shows the total heat loss to the walls, both the walls included in the LES and the estimated total if the side walls were included. The latter increases the heat losses by $13 \%$, but does not qualitatively change anything.

When increasing the ER, about half of the additional heat release is lost to wall heat transfer. The increased wall heat transfer $q_{w}$ is caused by a combination of factors, including lower convection velocity, faster mixing (which brings hot gases to the walls), and higher local heat release. As noted above in reference to Fig. 13, both the increased $q_{w}$ and the (minorly) decreased wall friction $\tau_{w}$ will have a stabilizing effect on the shock-train, as they will drive the flow away from choking conditions. Of the two, the increased wall heat transfer will have the dominant effect. Fig. 21 shows, however, that this increased $q_{w}$ is far from sufficient to accommodate the elevated heat release at higher ERs. We thus conclude that the redistribution of the cross-sectional heat release by the shock-train as described earlier also plays a significant role in allowing it to stabilize in the combustor.

Finally, we look to the thrust-generating potential of the combustor at various ERs. Since the LES domains used in this study only cover the combustor and the beginning of the nozzle, the thrust can not be computed directly. Instead we compute a surrogate quantity (the "potential thrust") by taking the cross-sectionally averaged state at the combustor exit and expanding it isentropically to an assumed ambient pressure of $2 \mathrm{kPa}$; this gives a gross thrust from an idealized nozzle. The ram drag is calculated from the inflow mass flux and the known free-stream velocity, while the frictional drag from the isolator and combustor are taken from the LES. The results are shown in Fig. 22. The first point to note is that the HyShot II, as might be expected, is an extremely poor thrust-producing device, with the net thrust being negative at all ERs considered (though only barely so at ER=0.524). The thrust increases monotonically with ER, and the slope of the curve is hardly altered by the development of the combustor shock-train. This suggests that the enhanced combustion efficiency is counterbalancing the increased losses associated with the combustor shock-train.

As one last note, returning to Fig. 13 we observe that the shock-train has an almost instantaneous effect on accelerating $\mathrm{H}_{2} \mathrm{O}$ production, but further downstream the source term decreases substantially. Thus, the introduction of a combustor shock-train into the flow could potentially allow for shorter combustionchamber lengths (with corresponding reductions in frictional and heat losses) with only a minimal compromise in the combustion efficiency.

\section{Summary}

This paper describes work toward predictive large eddy simulations (LES) of the reacting flow in the HyShot II scramjet combustor, specifically focused on the solutions near the limit of operability. The high Reynolds number makes traditional LES completely impractical, and thus a wall-model for the innermost portion of the boundary layers is needed. The combustion chemistry is parametrized through a flameletbased modeling approach, with a presumed PDF for the mixture fraction. The strength of this modeling approach is the direct resolution of the flame micro structure, the fact that turbulence-induced subgrid fluctuations of the mixture fraction are accounted for, and the alleviation of any combustion-induced 
stiffness on the time step; the main weaknesses (in its current form) are the inability to accurately predict auto-ignition (in the steady flamelet framework) and the lack of feedback from flow-induced changes in pressure and enthalpy on the detailed chemistry of minor species. These issues are part of ongoing research; however, it must be noted that the main objective of the present study is to predict the pressurerise in the combustor, rather than minor species concentrations. The predicted pressure-rise is within the experimental bounds, and shows reasonable grid-convergence.

With this verification/validation study in hand, the simulation methodology is then used to investigate the flow for higher equivalence ratios (ERs). This is done by keeping the shock tunnel conditions fixed while increasing the total pressure of the fuel stream in finite steps. The LES and the experiments both show steady state solutions with essentially linear pressure profiles for ERs $\lesssim 0.38$, and the appearance of a "combustor shock-train" near the end of the combustor for ERs $\gtrsim 0.40$ and 0.39, respectively (note that no LES has been run at $\mathrm{ER}=0.39)$. This combustor shock-train is similar to the normal shock-trains that appear in the isolator during dual-mode operation, but here coincides spatially with the combustion and heat release. The combustor shock-train appears to be rather robust, in that it appears in both LES and experiments despite them having rather different initial transients toward that final state. In the LES, a stable combustor shock-train occurs for $0.40 \lesssim \mathrm{ER} \lesssim 0.52$. The available thrust increases up to ER $\approx 0.52$, and the majority of the combustion completes over a shorter distance, suggesting that operation with a combustor shock-train might in fact be desirable in practice.

\section{Acknowledgments}

This work was supported by the Department of Energy Predictive Science Academic Alliance Program (PSAAP; grant DE-FC52-08NA28614). Computing resources were partially provided through the American Recovery and Reinvestment Act of 2009, through the NSF-funded Certainty cluster. The work has benefitted from stimulating discussions and insightful comments by Mirko Gamba and Carlo Scalo, and from 2D RANS results for the inflow condition by Michael Emory.

\section{References}

[1] E. Curran, W. Heiser, D. Pratt, Fluid phenomena in scramjet combustion systems, Annu. Rev. Fluid Mech. 28 (1996) 323-360.

[2] E. T. Curran, F. D. Stull, USAF Tech. Rep. RTD-TDR-63-4097, Wright-Patterson AFB, 1964.

[3] W. Heiser, D. Pratt, Hypersonic airbreathing propulsion, AIAA Education Series, AIAA, Washington, DC, 1994.

[4] K. Matsuo, Y. Miyazato, H.-D. Kim, Shock train and pseudo-shock phenomena in internal gas flows, Prog. Aero. Sci. 35 (1999) 33-100. 
[5] G. Y. Anderson, C. R. McClinton, J. P. Weidner, Scramjet performance, in: E. Curran, S. Murphy (Eds.), Scramjet Propulsion, volume 189 of Progress in Astronautics and Aeronautics, AIAA, 2000, pp. 369-439.

[6] J. Fulton, J. R. Edwards, H. A. Hassan, R. Rockwell, C. Goyne, J. McDaniel, C. Smith, A. Cutler, C. Johansen, P. M. Danehy, T. Kouchi, Large-eddy/Reynolds-averaged Navier-Stokes simulations of a dual-mode scramjet combustor, AIAA Paper 2012-0115, 2012.

[7] C. Fureby, LES for supersonic combustion, AIAA Paper 2012-5979, 2012.

[8] M. K. Smart, N. E. Hass, A. Paull, Flight data analysis of the HyShot 2 scramjet flight experiment, AIAA J. 44 (2006) 2366-2375.

[9] A. D. Gardner, K. Hannemann, J. Steelant, A. Paull, Ground testing of the HyShot supersonic combustion flight experiment in HEG and comparison with flight data, AIAA Paper 2004-3345, 2004.

[10] K. Hannemann, J. Martinez Schramm, S. Karl, J. Steelant, Experimental investigation of different scramjet hydrogen injection systems, in: Proc. 6th European Symposium on Aerothermodynamics for Space Vehicles, ESA-SP-659, 2009.

[11] K. Hannemann, S. Karl, J. Martinez Schramm, J. Steelant, Methodology of a combined ground based testing and numerical modelling analysis of supersonic combustion flow paths, Shock Waves 20 (2010) 353-366.

[12] S. J. Laurence, H. Ozawa, D. Lieber, J. Martinez Schramm, K. Hannemann, Investigation of unsteady/quasi-steady scramjet behavior using high-speed visualization techniques, AIAA Paper 2012-5913, 2012.

[13] S. J. Laurence, S. Karl, J. Martinez Schramm, K. Hannemann, Transient fluid-combustion phenomena in a model scramjet, J. Fluid Mech. 722 (2013) 85-120.

[14] S. J. Laurence, S. Karl, K. Hannemann, Experimental and numerical investigation of the HyShot II flight experiment, in: ISSW30, Madison, USA, 2013.

[15] S. J. Laurence, D. Lieber, J. Martinez Schramm, K. Hannemann, J. Larsson, Incipient thermal choking and stable shock-train formation in the heat-release region of a scramjet combustor. Part I: Shock-tunnel experiments, Combust. Flame (2014) accepted.

[16] S. Karl, K. Hannemann, J. Steelant, A. Mack, CFD analysis of the HyShot supersonic combustion flight experiment configuration, AIAA Paper 2006-8041, 2006.

[17] S. Karl, K. Hannemann, A. Mack, J. Steelant, CFD analysis of the HyShot II scramjet experiments in the HEG shock tunnel, AIAA Paper 2008-2548, 2008. 
[18] S. Karl, K. Hannemann, A. Mack, J. Steelant, CFD analysis of unstart characteristics of the HyShot II scramjet configuration in the HEG shock tunnel, AIAA Paper 2011-2309, 2011.

[19] S. Karl, Numerical Investigation of a Generic Scramjet Configuration, Ph.D. thesis, Technische Universität Dresden, Dresden, Germany, 2011.

[20] S. Karl, S. J. Laurence, J. Martinez Schramm, K. Hannemann, CFD analysis of unsteady combustion phenomena in the HyShot-II scramjet configuration, AIAA Paper 2012-5912, 2012.

[21] R. Pecnik, V. E. Terrapon, F. Ham, G. Iaccarino, H. Pitsch, Reynolds-averaged Navier-Stokes simulations of the HyShot II scramjet, AIAA J. 50 (2012) 1717-1732.

[22] C. Fureby, M. Chapuis, E. Fedina, S. Karl, CFD analysis of the HyShot II scramjet combustor, Proc. Comb. Inst. 33 (2011) 2399-2405.

[23] M. Chapuis, E. Fedina, C. Fureby, K. Hannemann, S. Karl, J. Martinez Schramm, A computational study of the HyShot II combustor performance, Proc. Comb. Inst. 34 (2013) 2101-2109.

[24] M. Berglund, C. Fureby, LES of supersonic combustion in a scramjet engine model, Proc. Comb. Inst. 31 (2007) 2497-2504.

[25] A. W. Vreman, An eddy-viscosity subgrid-scale model for turbulent shear flow: Algebraic theory and applications, Phys. Fluids 16 (2004) 3670-3681.

[26] J. Larsson, B. Gustafsson, Stability criteria for hybrid difference methods, J. Comput. Phys. 227 (2008) 2886-2898.

[27] J. Larsson, Effect of shock-capturing errors on turbulence statistics, AIAA J. 48 (2010) 1554-1557.

[28] E. F. Toro, M. Spruce, M. Speares, Restoration of the contact surface in the HLL-Riemann solver, Shock Waves 4 (1994) 25-34.

[29] Y. Khalighi, J. W. Nichols, S. K. Lele, F. Ham, P. Moin, Unstructured large eddy simulation for prediction of noise issued from turbulent jets in various configurations, AIAA Paper 2011-2886, 2011.

[30] M. Emory, V. Terrapon, R. Pecnik, G. Iaccarino, Characterizing the operability limits of the HyShot II scramjet through RANS simulations, AIAA Paper 2011-2282, 2011.

[31] M. Klein, A. Sadiki, J. Janicka, A digital filter based generation of inflow data for spatially developing direct numerical or large eddy simulations, J. Comput. Phys. 186 (2003) 652-665.

[32] A. Saghafian, High-fidelity simulations and modeling of compressible reacting flows, $\mathrm{PhD}$ thesis, Department of Mechanical Engineering, Stanford University, 2014.

[33] C. D. Pierce, P. Moin, Progress-variable approach for large-eddy simulation of non-premixed turbulent combustion, J. Fluid Mech. 504 (2004) 73-97. 
[34] V. Sabelnikov, B. Deshaies, L. F. F. Da Silva, Revisited flamelet model for nonpremixed combustion in supersonic flows, Combust. Flame 114 (1998) 577-584.

[35] J. R. Edwards, J. A. Boles, R. A. Baurle, Large-eddy/Reynolds-averaged Navier-Stokes simulation of a supersonic reacting wall jet, Combust. Flame 159 (2012) 1127-1138.

[36] Z. Hong, D. F. Davidson, R. K. Hanson, An improved $\mathrm{H}_{2} / \mathrm{O}_{2}$ mechanism based on recent shock tube/laser absorption measurements, Combust. Flame 158 (2011) 633-644.

[37] A. Saghafian, V. E. Terrapon, H. Pitsch, An efficient flamelet-based combustion model for compressible flows, Combust. Flame (2014) accepted.

[38] S. Kawai, J. Larsson, Wall-modeling in large eddy simulation: length scales, grid resolution and accuracy, Phys. Fluids 24 (2012) 015105.

[39] D. B. Helmer, L. M. Campo, J. K. Eaton, Three-dimensional features of a Mach 2.1 shock/boundary layer interaction, Exp. Fluids 53 (2012) 1347-1368.

[40] L. M. Campo, D. B. Helmer, J. K. Eaton, PIV investigation of spanwise variation in incident shock boundary layer interactions, in: Turbulence and Shear Flow Phenomena 8, 2013.

[41] I. Bermejo-Moreno, J. Larsson, L. Campo, J. Bodart, R. Vicquelin, D. Helmer, J. Eaton, Wallmodeled large eddy simulation of shock/turbulent boundary-layer interaction in a duct, in: Annu. Res. Briefs, Center for Turbulence Research, 2011, pp. 49-62.

[42] J. Larsson, I. Bermejo-Moreno, S. K. Lele, Reynolds- and Mach-number effects in canonical shockturbulence interaction, J. Fluid Mech. 717 (2013) 293-321. 

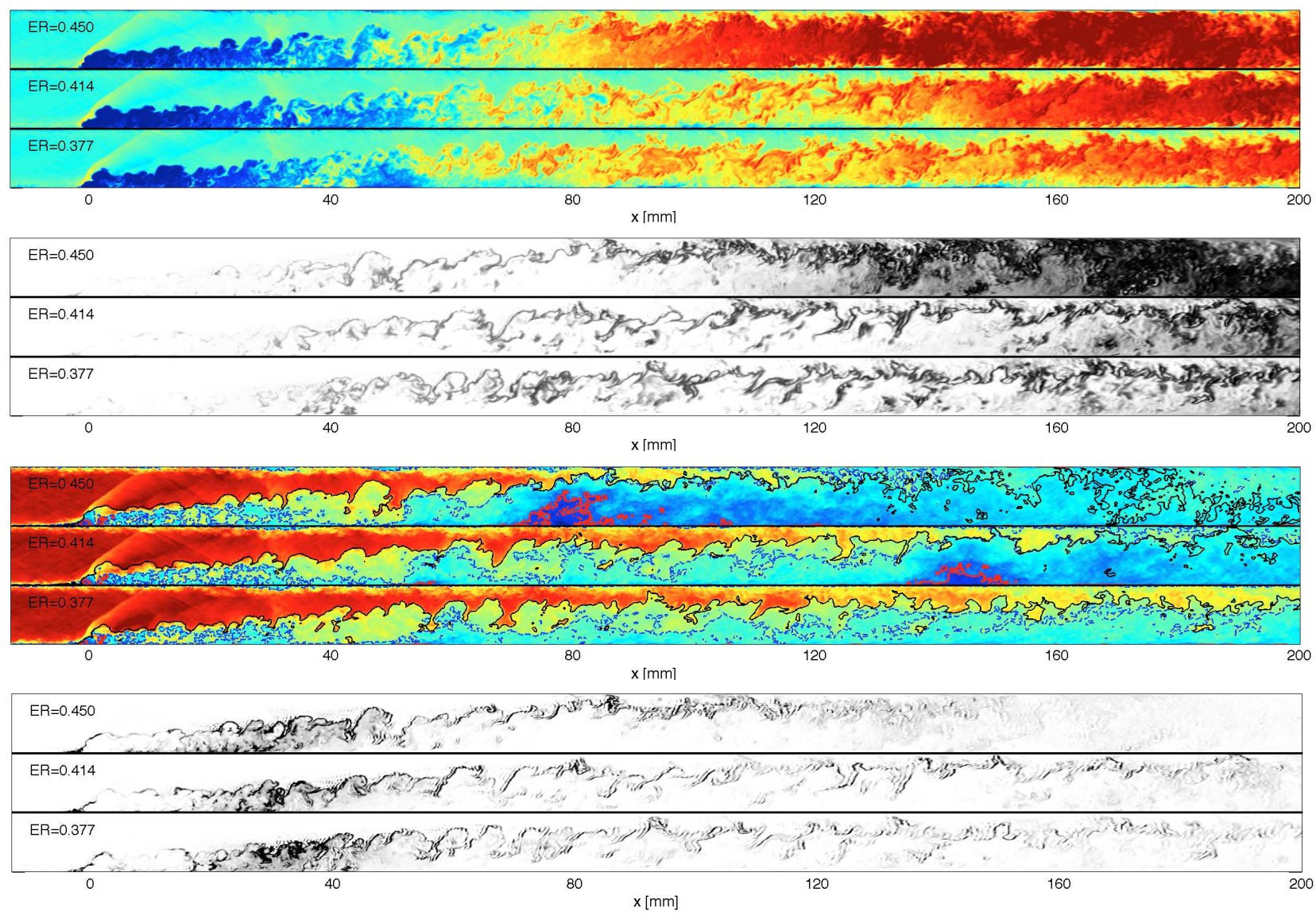

Figure 14: Contours in a plane through the fuel injector, from $x=-15 \mathrm{~mm}$ to $x=200 \mathrm{~mm}$ (i.e., not quite the full length of the combustor). Colored from low (blue or white) to high (red or black) values. From top to bottom: temperature (300 K to $3000 \mathrm{~K})$; $\mathrm{OH}$ mass fraction (0 to 0.02$)$; streamwise Mach number $u / c(-0.5$ to 2.7$) ; \mathrm{H}_{2} \mathrm{O}$ production rate $\dot{\omega}_{\mathrm{H}_{2} \mathrm{O}}(0$ to 4000 $\left.\mathrm{kg} /\left(\mathrm{m}^{3} \mathrm{~s}\right)\right)$. In the third figure is also shown the stoichiometric line $(Z=0.028$; solid thin line), the sonic line (dashed thin line), and the line separating reverse flow $(u<0$; red solid thick line). 

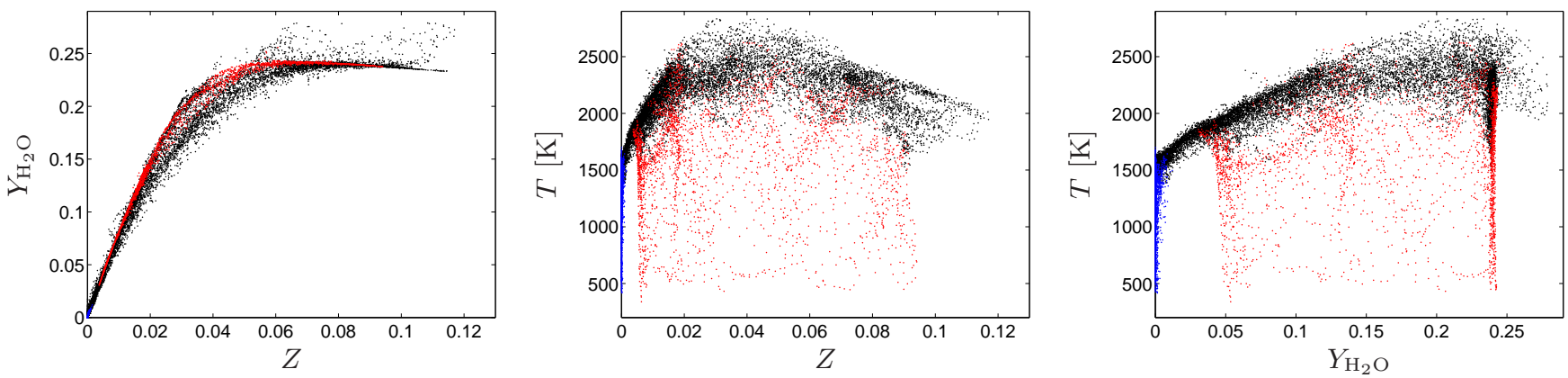

Figure 15: Scatter plots at $x=115 \mathrm{~mm}$ for $\mathrm{ER}=0.377$. Points within $0.5 \mathrm{~mm}$ of the upper wall are colored blue $(Z \approx 0$, some reaching low $T$ ), points within $0.5 \mathrm{~mm}$ of the lower wall are colored red (equilibrium $Y_{\mathrm{H}_{2} \mathrm{O}}$, some reaching low $T$ ), while the remaining points are colored black (cloud of points around equilibrium $Y_{\mathrm{H}_{2} \mathrm{O}}$ and $T$ ).

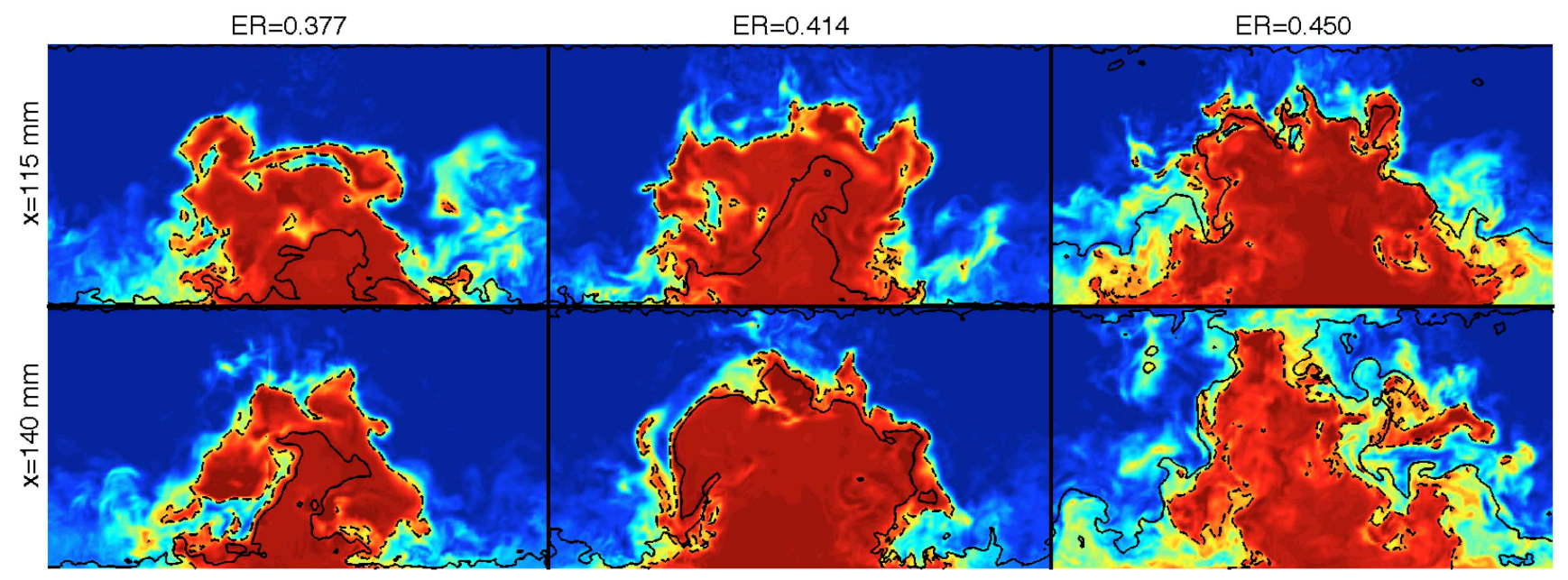

Figure 16: Contours of instantaneous $\mathrm{H}_{2} \mathrm{O}$ mass fraction in cross-sectional $y z$-slices, colored from 0 to 0.25 . ERs of 0.377 (left column), 0.414 (middle column) and 0.450 (right column). Also shown are the stoichiometric line (dashed line) and the sonic line (solid line). Top row: $x=115 \mathrm{~mm}$. Bottom row: $x=140 \mathrm{~mm}$. Note that the combustor shock-train starts at $x_{s} \approx 140 \mathrm{~mm}(\mathrm{ER}=0.414)$ and $x_{s} \approx 75 \mathrm{~mm}(\mathrm{ER}=0.450)$, respectively. 


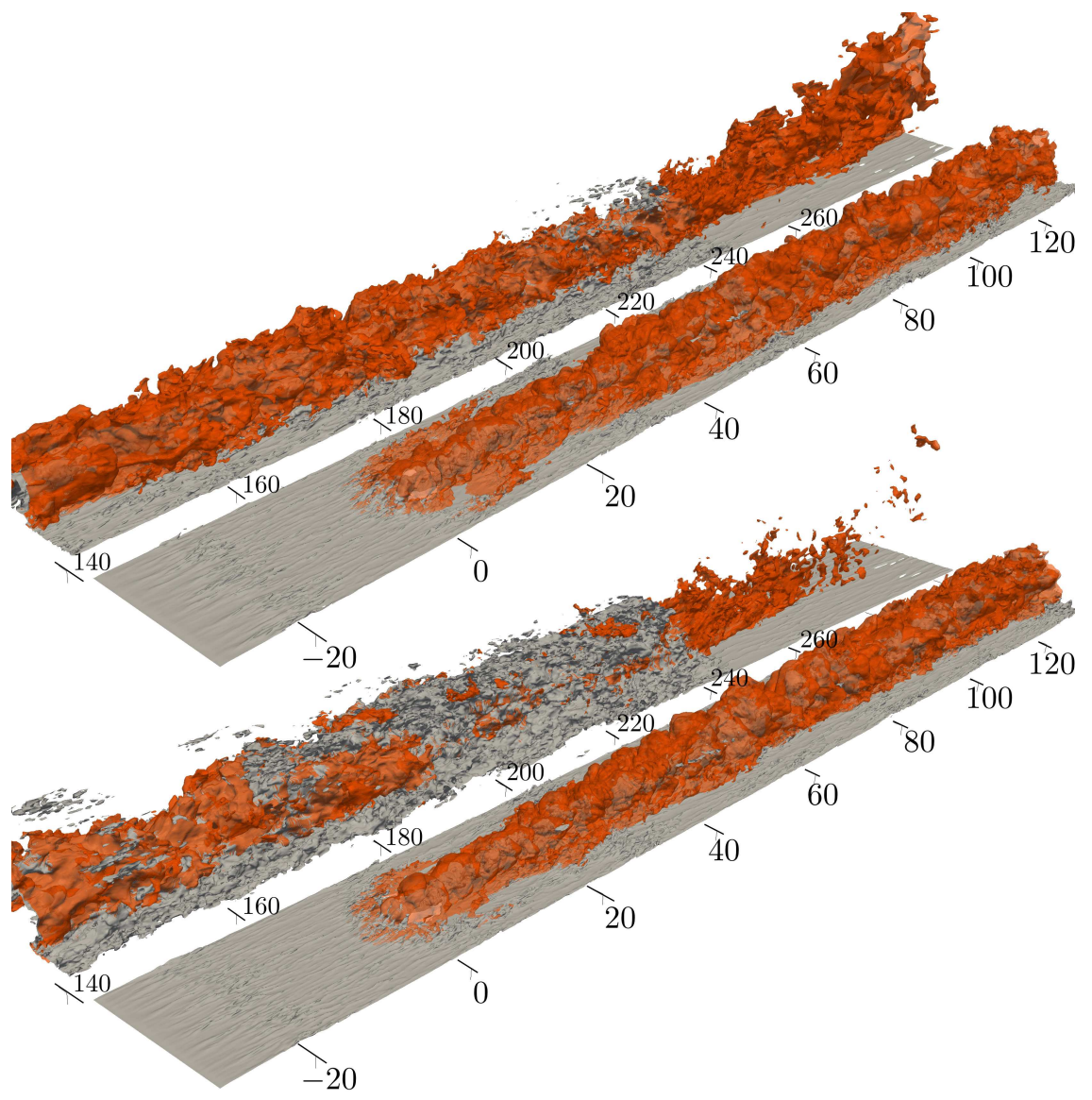

Figure 17: Isosurfaces of stoichiometric mixture $Z=0.028$ (orange) and sonic flow $M=1$ (gray), for ER=0.377 (top) and $\mathrm{ER}=0.414$ (bottom). To ease visualization, the combustor is split in two at $x \approx 135 \mathrm{~mm}$ in both cases. 


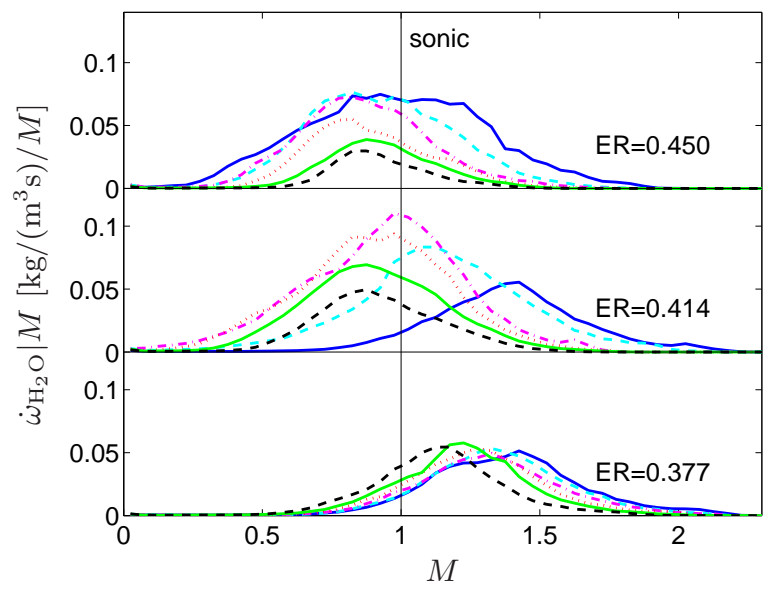

Figure 18: Source term of $\mathrm{H}_{2} \mathrm{O}$ per unit length conditioned on the Mach number $M$ at different streamwise locations (the area under each curve is the production rate per unit length). Shown at different spatial locations $x=115 \mathrm{~mm}$ (solid), 140 $\mathrm{mm}$ (dashed), $150 \mathrm{~mm}$ (dash-dotted), $170 \mathrm{~mm}$ (dotted), $190 \mathrm{~mm}$ (solid), and $215 \mathrm{~mm}$ (dashed). Note that the combustor shock-train starts at $x_{s} \approx 140 \mathrm{~mm}(\mathrm{ER}=0.414)$ and $x_{s} \approx 75 \mathrm{~mm}(\mathrm{ER}=0.450)$, respectively. Different ERs shown with vertical offset.

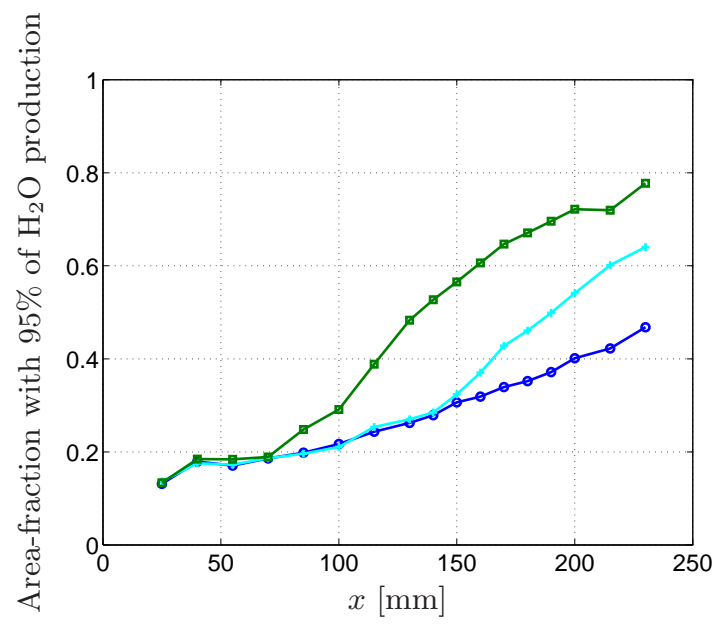

Figure 19: Fraction of the cross-sectional area over which 95\% of the $\mathrm{H}_{2} \mathrm{O}$ production occurs. ERs of 0.377 (circles), 0.414 (pluses), and 0.450 (squares). Note that the combustor shock-train starts at $x_{s} \approx 140 \mathrm{~mm}(\mathrm{ER}=0.414)$ and $x_{s} \approx 75 \mathrm{~mm}$ $(\mathrm{ER}=0.450)$, respectively. 


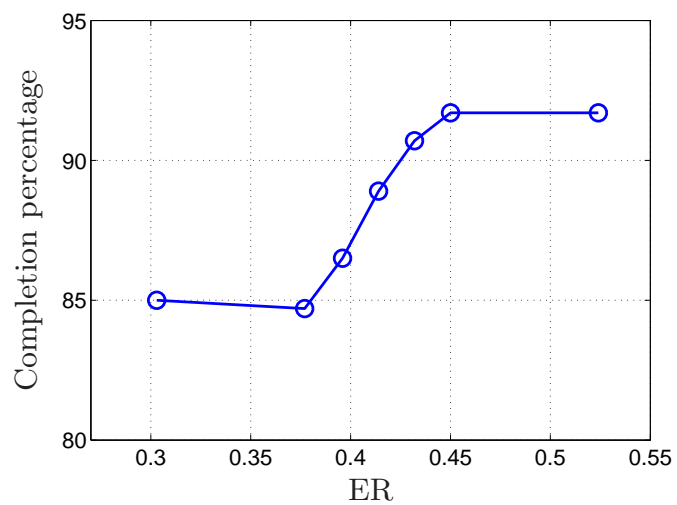

Figure 20: Combustion efficiency defined as the percentage of $\mathrm{H}_{2}$ that has formed $\mathrm{H}_{2} \mathrm{O}$ by the exit of the domain.

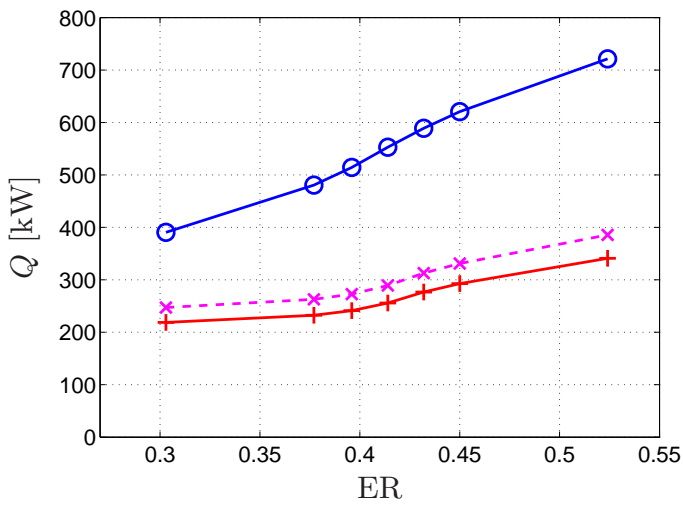

Figure 21: Heat balance in the combustor (for full width, i.e., 4 fuel injectors). Chemical heat release (circles) compared with heat losses to the horizontal walls (pluses). The heat loss to the side walls is estimated by assuming a similar heat flux; the resulting estimated total heat loss is shown with a dashed line. 


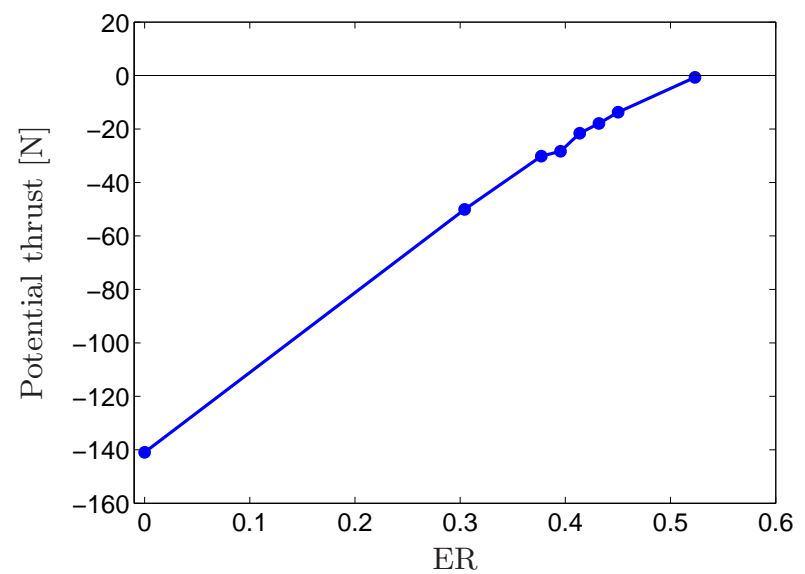

Figure 22: Potential (maximum available) thrust at different equivalence ratios (ER), evaluated by assuming isentropic nozzle expansion to $2 \mathrm{kPa}$ ambient pressure. The wall friction from the LES is included in the thrust, and the ram drag from the inlet is estimated from the mass flux and the free-stream conditions. The thrust is given for the full HyShot II geometry, i.e., for 4 fuel injectors. 